This report was ptepared as an account of work sponsored by an agency of the Uniled Stales Government. Neither the United States Government nor any agency thereof, nor any of their employees, makes any warranty, express or implied, or assumes any legal liability or responsibility for the accuracy, compleieness, or usefuliness of any information, apparatus, product, ot process disclosed, or represents that its use would not infringe privately owned rights. Reference herein to any specific commercial product, process, or service by trade name, trademark. manufacturer, or otherwise does not necessarily constitute or imply its endorsement, recommendation, of favoring by the United States Government ar any agency thereof. The views and opinions of authors expressed hercin do not recessurily state or relect those of the United States Government or any agency thereof.

\title{
Transport Simulations of a Density Limit in Radiation-Dominated Tokamak Discharges - Profile Effects
}

\author{
D. P. Stotler \\ Plasma Physics Laboratory \\ Princeton University \\ Princeton, New Jersey 08543
}

\begin{abstract}
The density limit observed in tokamak experiments is thought to be due to a radjative collapse of the current channel. A transport code coupled with an MHD equilibrium routine is used to determine the detailed, self-consistent evolution of the plasma profiles in tokamak discharges with radiated power close to or equalling the input power. The present work is confined to ohmic discharges in steady state. It is found that the shape of the density profile can have a significant impact on the variation of the maximum electron density with plasma current. Analytic calculations confirm this result.
\end{abstract}




\section{Introduction}

The limit on density observed in present tokamak experiments is not of much interest except as a parameter space boundary that is to be avoided. However, in a burning plasma it represents a linit to the amount of fusion power that can be produced; that is. it restricts the value of $n \tau_{E}$ ( $n$ is the plasma density, $\tau_{E}$ is the energy confinement time) that can be achieved. In fact, transport simulations of two recent reactor designs ${ }^{d}$ and the Compact Ignition Tokamak ${ }^{2,3}$ (CIT) do not predict ignition with an L-modet scaling for $\tau_{E}$ when the density is required to remain below that predicted by present expressions for the density limit. Several formulas for the density limit are now in use, and most match recent data very well. However, when extrapolated to high fields and significantly noncircular cross sections, their predictions vary considerably. In an a:tempt to reduce the uncertainty in the density limit scaling law, we will use the 1-1/2-D BALDUR ${ }^{5,6}$ transport code to simulate high density tokamak discharges. Hopefully, this will lead to a model of the density limit with a predictive capability. If the procedure is successful, the resulting model could be used to optimize the design of future ignition devices. In this paper we will describe the first step in the development of such a simulation model and will show results for ohmic discharges. Later work will include detailed comparisons with experiments.

The first scaling law for the maximum density in tokamaks was proposed by Murakami et al. ${ }^{7}$ They noted that $\bar{n}_{e, \text { mor }} \propto B_{T} / R$, where $\overline{n_{e}}$ is the lineaveraged electron density, $B_{T}$ is the toroidal magnetic field, and $R$ is the plasma major radius. This scaling was later refined to include a dependence on the plasma current through the safety factor, $q$; namely, ${ }_{\overline{n_{e}, \max }} \propto B_{T} / R q$. A density limit expressed in this form is often referred to as the Hugill limit. The Murakami limit is then the maximum density for all values of the plasma current.

It is not clear how to extend the scaling law for the Hugill limit to include tokamaks having elongated cross sections due to the wide variety of analytic expressions for $q$ and its cylindrical equivalent, qul. Greenwald has recently made an attempt to consolide the data from machines of various shapes and sizes. ${ }^{8,10} \mathrm{He}$ concluded that a tokamak with a high power density, Jow impurity level, and efficient central fuelling is limited to a density $\bar{\pi}_{e, G}=$ $\mathrm{r} J \times 10^{20} \mathrm{~m}^{-3}$, where $\kappa$ is the plasma elongation and $J$ is the average plasma 
current density (in $M A / \mathrm{m}^{2}$ ). Compared to present Hugill-type scalings, this expression tends to be more favorable for highly elongated reac:or designs.

Untortunately, the range of $\kappa$ covered in the present data base is not sufficient to rule out clearly the Hugill-type scalings. For example, the maximum density observed in the Joint European Torus $(\mathrm{JET})^{11}$ is $\bar{n}_{e, \max }=2 B_{T} / R q_{e}$, where $\varphi_{e}=\bar{j} \omega^{2} \kappa B_{T} / R I_{p}$ is referred to as the engineering salety factor. Nute that we express $B_{T}$ in $\mathrm{T}, a$ (the plasma minor radius) and $R$ in $\mathrm{m}$, and $I_{\mathrm{p}}$ (the plasma current) in MA. Given the uncertainties in the experimental measurcments and the limitation to $\kappa<1.7$ in the JET device, it is difficult to discern between this and the Greenwald expression. Yet, the values they predict differ significantly at elongations of $\kappa \geq 2$, typical of reactor designs. The more theoretical approach of transport simulation could provide one means of bridging this gap in the data base.

Most of the explanations proposed to date for the density limit rely upon impurity radiation-induced profile changes as the primary cause of the disruption. ${ }^{12-17}$ However, one of the conclusions drawn by Greenwald is that the maximum density obtainable for a given current is determined not by radiation, but by a deterioration of the particle confinement in the plasma core. Due to the lack of detailed models for this process, transport codes can do little to clarify the situation except to provide a means for carrying out the arduous task of preparing complete simulations of particular discharges. We can, however, examine with relative ease many of the consequences of impurity radiation. Therefore, we confine our attention in the present work to discharges dominated by impurity line radiation.

The model used for this radiation is of great importanr... Wost of the theoretical work carried out so far has employed the coronal equilibrium model ${ }^{18}$ due to its relative simplicity. However, the model is known to yield radiated power levels that are far below those found in actual experiments. ${ }^{19}$ On the other hand, by using an empirically determined scaling for the radiation in JET, Campbell et al. ${ }^{20}$ were able to derive a reasonable expression for the density limit simply by balancing the radiated power and the total input power. There are limitations on the parameters for which this expression is applicable; hence, there is a need for more theoretical input. Computer codes capable of accurately modelling the radiation in systems deviating from coronal equilibrium are presently being used in the analysis of tokamak data. 21 Hopefully, these same techniques can be applied to studies of the density 
limit.

With a 1-1/2-D transport code, we can investigate self-consistent. timedependent profile effects. We have complete freedom in the specification of transport models and plasma compositions. Plasma fuelling by gas puffing and pellet injection can both be modelled. In addition to ohmic heating, auxiliary input power from neutral beam injection or other sources can be included. By coupling the transport code to an equilibrium code, we can investigate MHD effects in detail. For example, sawtooth oscillations and tearing modes (currently being installed in the BALDUR code ${ }^{22}$ ) will have a significant impact on the discharges we investigate.

The present work is limited to steady-state simulations of ohmic discharges; a TFTR geometry is used. ${ }^{23}$ In particular, we focus on density profile effects. The most important difference between flat and peaked density profiles is that, for a given volume-averaged density, a fat profile has a higher density near the edge. It is in this low temperature edge segion that the impurity line radiation (proportional to the local product of the electron density and the impurity density) peaks. The higher density in the flat profile case leads to a greater contribution to the overall radiated power. Hence, flat profile cases are restricted to lower average densities than peaked profile discharges with the same input power.

What is more surprising is that in simulations with centrally peaked density profiles (edge density much less than central density) there is a significant virjation in the critical Mlurakami parameter with $q_{c y l}$, as observed experimentally (i.e., the Hugill limit). On the other hand, with flat profiles (edge density slightly less than central density), the critical Murakami parameter is almost independent of $g_{c y l}$. We show analytically that these results are a consequence of the effect changing $q_{c y l}$ has on the sawtooth mixing radius, (in our model) the electron temperature profile, and safety factor profile.

In Sec. 11, we specify the simulation model we will be using. We give our numerical results in Sec. III. The analytic calculations will be described in Sec. IV. Finally, a discussion and summary is presented in Sec. V.

\section{Simulation Model}

We now describe briefly our transport code and discuss the models and assumptions used in the simulations. We employ the $1-1 / 2-D$ BALDLR. 
transport code; details on it have been given elsewhere. ${ }^{5.6}$ It integrates in time a set of flux-surface-averaged transport equations on a radial grid of surfaces of constant toroidal flux. Their shape is provided by a set of moments equilibrium routines that are interfaced with the transport code. The transport equations solved describe the diffusion of density, energy, and magnetic. field:

$$
\begin{aligned}
& \frac{\partial}{\partial t}{ }_{\xi}\left(n V^{\prime \prime}\right)=-\frac{\partial}{\partial \xi}\left(V^{\prime}\left\langle\Gamma_{p} \cdot \nabla \xi\right\rangle-n V^{\prime \prime} \dot{\rho} \mid \rho^{\prime}\right)+V^{\prime}\langle S\rangle, \\
& \left.\frac{3}{2} \frac{\partial}{\partial t}\right|_{\xi}\left(n T V^{\prime \prime}\right)=-\frac{\partial}{\partial \xi}\left(V^{\prime \prime}\left(\eta_{\rho} \cdot \nabla \xi\right)-\frac{3 \pi \Gamma l^{\prime \prime} \dot{\rho}}{\rho^{\prime}}\right) \\
& -V^{\prime}\langle Q\rangle-n T\left[\dot{V}-\left(\dot{\rho} l^{\prime \prime} / \rho^{\prime}\right)^{\prime}\right] . \\
& \left.\frac{\partial}{\partial t}\right|_{\xi}\left(B_{p} \rho^{\prime}\right)=\frac{\partial}{\partial \xi}\left\{\frac { \eta } { I ( 1 / R ^ { 2 } ) } \left[\frac{l^{2}}{\mu_{0} V^{\prime}} \frac{\partial}{\partial \xi}\left(\frac{l^{\prime \prime} \rho^{\prime} B_{p}}{I}\left(\frac{\left.\nabla \xi\right|^{2}}{R^{2}}\right)\right)\right.\right. \\
& \left.\left.-\frac{\left\langle J_{\text {beam }} \cdot B\right\rangle}{R_{\text {OREF }}}-\frac{\left\langle J_{\text {boot }} \cdot B\right\rangle}{R_{0 R E F}}\right]-\dot{\rho} B_{p}\right\} \text {. }
\end{aligned}
$$

There are separate equations for the density, $n(\xi, t)$, of each ionic species. The electron density is computed from quasi-neutrality. Separate energy equations are solved for jons and electrons; the temperatures, $T(\xi, t)$, of all ionic species are taken to be equal. The quantity appearing in Eq. (3) is actually a normalized poloidal flux gradient:

$$
B_{p} \equiv \frac{\partial \psi_{\text {pol }} / \partial \rho}{2 \pi R_{0 R E F}}
$$

In the limit of a circular cylinder geometry, this $B_{p}$ reduces to the actual poloidal magnetic field. Note that $\rho$ is a flux-surface label defined by $\rho \equiv$ $\left(\psi_{\mathrm{cor}} / \pi B_{O R E F}\right)^{1 / 2} ; B_{O R E F}$ and $R_{O R E F}$ are a fixed reference magnetic field and major radius, respectively. The equations have been transformed into a coordinate system with arbitrary flux surface label $\xi$. The toroidal (poloidal) fluxes are denoted by $\psi_{\text {tor }}\left(\psi_{\mathrm{p} \alpha}\right)$. The volume inside flux surface $\xi$ is $V(\xi, t)$; $V^{\prime}=\partial V / \partial \xi$. An overdot indicates a derivative with respect to time. The particle and heat fluxes are designated as $\boldsymbol{\Gamma}_{\rho}$ and $\boldsymbol{q}_{\boldsymbol{\rho}}$. The subscript $\rho$ signifies that they are defined relative to surfaces of constant toroidal flux. Volume 
sources of particles (energy) are contained in $S(Q)$. Finally, in the magnetic diffusion equation. Eq. (3). $R$ is the major radius. $\eta$ is the plasma resistivity. and $I(\xi)=R B_{T}$, where $B_{T}$ is the toroidal magnetic field. We show for completeness contributions from beam-driven current $\left(J_{b e a m}\right)$ and bortstrap current $\left(J_{\text {boot }}\right.$ ), although we do not make use of either in these simulatiuns. In all cases. the angle brackets denote a fux-iurface average.

IVe will keep the expressions for the fluxes relatjvely simple in order to focus on the effects of radiation in the power balance. The expression we use for the heat fux is

$$
\left\langle\boldsymbol{q}_{\rho} \cdot \nabla \xi\right\rangle_{j}=\left(-\chi_{j} \frac{\partial}{\partial \xi}\left\langle\frac{3}{2} n_{j} T_{j}\right\rangle\langle|\nabla \xi|\rangle \div \frac{3}{2} T_{j}\left(\Gamma_{p} \cdot \nabla \xi\right\rangle_{j}\right) \frac{\left\langle, \nabla \xi \xi^{2}\right\rangle}{\left\langle\nabla \xi_{i}\right\rangle},
$$

where the subscript $j$ denotes electron or ion thermal fux. In this particular version of BALDLR, the flux-surface label, $\xi$, is taken to be the square root of the normalized toroidal flux, $\xi=\left(\dot{\psi}_{\text {tor }} / \psi_{\text {tor,a }}\right)^{1 / 2}$, where $\psi_{t a r, a}$ is the toroidal flux at the plasma boundary.

The thermal diflusivities are based upon the INTOR model. ${ }^{29}$ They should give rise to a neo-Alcator type scaling for the energy confinement time, as is appropriate for an ohmic discharge. One could argue that this is not suitable at the highest densities where a saturation of the energy confinement time with density is usually observed. ${ }^{25}$ In the interest of simplicity, however. we use the following model for all of the simulations discussed here. We set

$$
\chi_{e}=2.6 / n_{e, 19}(\xi)+\chi_{e}^{N C},
$$

and

$$
\chi_{i}=1.3 / n_{c, 19}(\xi)+\chi_{i}^{C H} .
$$

The units on both are $\mathrm{m}^{2} / \mathrm{sec} ; n_{\mathrm{e}, 1}(\xi)$ is the local electron density in units of $10^{19} \mathrm{~m}^{-3}$. The neoclassical contribution computed by Chang and Hinton ${ }^{26}$ is included as $\chi_{i}^{C H}$. The neoclassical electron thermal diffusivity $\chi_{*}^{N C}$ is also included; see Ref. 5 for the detailed expression. The overall constants in Eqs. (6) and (7) have been chosen to yield reasonable central temperatures in a stead $y$-state, ohmic TFTR discharge $(\sim 2.5 \mathrm{keV}$, see for example Ref. 27$)$.

The particle flux of ionic species $j$ is written as

$$
\left\langle\Gamma_{\rho} \cdot \nabla \xi\right\rangle_{j}=\left(-D_{j} \frac{\partial n_{j}}{\partial \xi}\langle\nabla \xi \mid\rangle+n_{j} v_{j}-\Gamma_{j}^{\text {neo }}\right) \frac{\left(|\nabla \xi|^{2}\right\rangle}{\langle\mid \nabla \xi\rangle}
$$


and we assume

$$
\begin{aligned}
D & =0.5 \mathrm{~m}^{2} / \mathrm{sec} \\
v & =-2 D \mathrm{r} / \mathrm{a}^{2}
\end{aligned}
$$

$r$ is a generalization of the minor radial coordinate. Here, $r$ is defined as half of the width (the half-width) of a given flux surface on the midplane. It is thus a flux surface quantity. The neoclassical $f u x, \Gamma_{j}^{\text {neo }}$, includes the Ware pinch for the hydrogenic components. The complete expressions are given in Ref. 5 . These values for $D$ and $v$ are based loosely upon the TFTR simulations performed by Redi et al. ${ }^{27}$ We have increased $D$ by a factor of 5 to reduce the simulation time required for the profiles to reach steady state following an increase in density by gas puffing. We have also removed the impurity neoclassical contribution to $D$ so as to obtain centrally peaked impurity density profiles soon after impurity influxing. The resulting steadystate profiles are consistent with those found in simulations not employing impurity influxing.

This transport model leads to centrally peaked electron density profiles when combined with an edge density that is much less than the volume average. Flat density profiles are obtained by removing the anomalous invard pinch $(v=0)$ and raising the pedestal boundary condition for the hydrogenic species to a value close to the volume-averaged density.

The only source term we will describe here is the impurity line radiation. It appears as a sink in the electron energy balance equation. The other contributions to Eqs. (5) and (8) are given in Ref. 5. For low temperature ( $<2 \mathrm{keV}$ ), impure plasmas, the primary contribution to the radiative losses is from impurity line radiation. At low densities $\left(n_{e}<10^{22} \mathrm{~m}^{-3}\right)$, the plasma is transparent to its own radiation. Under these conditions, the electron collisional ionization rate can be balanced with the total recombination rate. Such systems are said to be in coronal equilibrium. ${ }^{18,28}$ The result is an expression for the radiated power per unit volume of the form

$$
p_{\text {rad }}=n_{e} n_{z} L_{2}\left(T_{e}\right)
$$

where $z$ indicates a particular impurity species. In addition to the function $L_{z}\left(T_{e}\right)$, one also obtains $\langle Z\rangle$ and $\left\langle Z^{2}\right\rangle$ as functions of the electron temperature, $T_{e}$, only. These are the average values of the impurity charge and 
its square in coronal equilibrium. In these simulations we will employ separately a high- $Z$ impurity, titanium, and a low- $Z$ impurity, carbon. We show in Figs. 1 and 2 plots of the formulas used ${ }^{18,28}$ for $L_{z}\left(T_{e}\right)$ and $\langle Z\rangle$.

In general, coronal equilibrium is thought to be a good approximation when the plasma is stable in time, the neutral density is small. and the transport time scales are long relative to ionization and recombination time scales. Although these conditions are not likely to hold for a typical TFTR discharge, we utilize the coronal equilibrium model in the present work for simplicity. In preparing for detailed comparisons with experimental results in future work. we intend to upgrade the BALDUR code to account for some of the expected deviations from coronal equilibrium. ${ }^{21}$

Sawtooth oscillations in BALDUR are modelled using a Kadomtser reconnection picture. ${ }^{29,30}$ Of course, for there to be a reconnection, the safety factor on axis must be less than unity. When it is, the code periodically flattens the density and temperature profiles out to the mixing radius; there is no (direct) alteration of the exterior portion of the plasma. The value of the mixing radius is computed from the Kadomtsev theory. ${ }^{30}$ It is typically 20 to $40 \%$ larger than the radius of the $q=1$ surface. Since detailed models for the periodic trigger mechanism of the sawtooth oscillations are not available, the user is reyuired to specity the period on input. For all of the simulations discussed here, it is fixed at $0.04 \mathrm{sec}$, typical of TFTR discharges exhibiting sawtouth oscillations. ${ }^{27}$

We wish to use this simulation model to fill out a Hugill diagram; that is, a plot of the maximum density attainable (normalized to $B_{T} / R$ ) as a function of $1 / q_{c y l}$. Here, we use $q_{c y l}=5 a^{2} B_{T} / R I_{p}$, where $a$ and $R$ are in $\mathrm{m}$, $B_{T}$ is in $\mathrm{T}$, and $I_{p}$ is in $\mathrm{MA}$. We start each simulation with parameters such that the radiated power is well below the ohmic input power. After allowing a short period of time for the plasma to come to steady state (typically 2 sec), we make a small change in some parameter. Following this, we ajlow another second of simulation time for the system to return to a steady state. The parameter is then altered again, and so on until the code fails. In most cases, these failures are the result of exceeding a specified number of time steps. The time steps shorten considerably as the profiles contract.

In order to isolate the effects due to varying sawtooth mixing radii, we choose to scan $\bar{n}_{e} R / B_{T}$ at constant $f_{e y d}$. We use one of two procedures to carry out the scan. The easiest is to decrease $I_{p}$ and $B_{T}$ at constant $I_{p} / B_{T}$, 
$R$, and $\overrightarrow{n_{e}}$. The other is to raise $\overrightarrow{n_{e}}$ by gas puffing. In order to keep $n_{z} / n_{e}$ constant, we also influx an appropriate amount of the impurity species. In this way, $Z_{\text {eff }}$ remains approximately constant. We define

$$
Z_{e f j} \equiv \frac{\sum_{j} n_{j}\left\langle Z_{j}^{2}\right\rangle}{n_{e}},
$$

where the sum is over all ionic species; their densities and charges are designated as $n_{j}$ and $\left\langle Z_{j}^{2}\right\rangle$, respectively. Wie will consider elsewhere the effects of allowing $n_{z} / n_{e}$ to vary. ${ }^{31}$

For all of the simulations described here, the injtial boundary conditions on the electron and ion temperatures at the plasma edge are of the pedestal type; typically, $T=20 \mathrm{eV}$. As the Murakami parameter, $\overline{n_{\mathrm{c}}} R / B_{T}$, is increased, the amount of radiation relative to the input power increases, making it more difficult to maintain the edge plasma. At some point, the tempecature of the computational zone just inside the boundary falls below the specified pedestal value. The presence of this positive gradient causes the code to switch to a zero energy flux boundary condition. Then, the edge temperature as a whole begins to fall, and the profile starts to contract.

We find that if $\overline{n_{e}} R / B_{T}$ is held fixed with a contracted profile, the plasma remains in a steady state. This mode of operation is reminiscent of the detached plasma experiments performed on TFTR. ${ }^{32,33}$ At the edge of the computational boundary, one finds that the total radiated power equals the input (ohmic) power. In other words, the conducted and convected powers at the edge are effectively zero since $T \simeq 0$ and $d T / d r \simeq 0$ there.

Because the present BALDUR code does not contain a detailed mechanism for identifying the conditions under which the plasma should disrupt, we must use a more arbitrary criterion for defining the density limit in our simulations.

Of course, the simplest limit is the smallest $\overline{n_{\varepsilon}} R / B_{T}$ for which the radiated power equals the input power. This would provide some interesting results, but would not tell the whole story. Namely, it is known that this is not a sufficient condition for a density limit disruption. ${ }^{16,20,32,33}$ Restricting ourselves to this limit would also prevent us from utilizing some of the key features of a 1-1/2-D transport code. Namely, we would not be able to inrestigate the process of forming the contracted profiles, nor would be able to consider separately their stability (through the built-in equilibrium data). 
The shape of the current profile in the vicinity of the $q=2$ surface determines the stability of the $m=2 . n=1$ tearing mode. This mode is thought to be instrumental in causing the observed disruption. ${ }^{16.20 .34}$ So, a reasonable disruption criteriun can be based upon the degree to which the profiles near $q=2$ are altered from their low radiation counterparts. For clarity, we chonse to define a limit as that value of the Murakami parameter for which $T_{e}=20 \mathrm{eV}$ at the $q=2$ surface. In this way, virtually no current is flowing outside of the $q=2$ surface..$^{16,20,35}$ It appears that a larger value for this critical temperature may be more appropriate. ${ }^{36}$

Another approach would be to plot the value of the internal inductance, $\ell_{i}$, against $q$ as the simulation evolves. Cheng, Furth, and Boozer ${ }^{37}$ have computed stability boundaries in this space for external kink and tearing modes in circular cross section cylinder geometry. Their results could be used to define a more theoretically precise disruption limit. However, without more calculations of the same sort for elongated geometries, it would be difficult to generalize this criterion to CIT and the various reactor designs. Furthermore, only recently has an appropriate diagnostic for $\ell_{i}$ been installed in BALDUR. Mlost of the simulations discussed in this paper were performed prior to this upgrade.

In the future, a saturated tearing mode package, ${ }^{22}$ currentiy being installed in BALDUR, will be utilized. By looking at the widths of the islands present, we might be able to arrive at a physically reasonable disruption limit. For now. we find it most convenient to define the density limit as occurring when $T_{e}=20 \mathrm{eV}$ at the $q=2$ surface. In addition to the above reasons, we prefer this procedure since it allows a precise definition of the critical Murakami parameter. However, it was necessary to use $T_{c}=200 \mathrm{eV}$ in the carbon impurity runs in order to compare uniformily all of the data. Some of these terminated prior to satisfying the original criterion.

In all of our simulations, we employ a TFTR geometry: $R=2.57 \mathrm{~m}$ and $a=0.82 \mathrm{~m}$. For our baseline parameters, we take $I_{p}=2.2 \mathrm{MA}, B_{T}=4.7$ $T$, and $\overline{n_{e}}=4.7 \times 10^{19} \mathrm{~m}^{-3}$. These values are typical of TFTR low-q, ohmic discharges. ${ }^{27}$ The actual plasma current, magnetic field, and electron density will vary from simulation to simulation.

The initial gas fill is mostly deuterium and hydrogen in a 9:1 ratio. In each run we use either titanium or carbon as the impurity species. When $\overline{n_{e}}$ is held fixed (or increased), gas puffing of the hydrogenic species in the 9:1 
ratio oflsets difusive losses. The cude is set up with a zero flux boundary condition on the impurity species so that their number js conserved in the absence of user-specifed influx.

\section{Results}

In Table I, we show the various parameters for each of the runs and the line-averaged electron density, plasma current, magnetic field, and Murakamu parameter at the time the disruption criterion is satisfied. All of the runs in the 02 and 03 series employ" a titanium impurity. Carbon is used in 05 and 06. Because the changes in the temperature affect the average charge state of the impurity; it is difficult to assign a precise value of $Z_{e / f}$ to each run. For the runs with titanium, $Z_{\text {eff }} \simeq 1.1-1.2$; with carbon, $Z_{\text {eff }} \simeq 4-5$. Less titanium can be tolerated as a result of the bigger and broader (in temperature) peaks in the $L\left(T_{e}\right)$ cutves shown in Fig. 1 .

The runs 02d, 02f, 02g, and 02h form a $q_{\text {esk }}$ scan with peaked density profiles. Similarly, 02i, 02j, 02k, and 021 form a $q_{c y}$ scan with that density Frofiles. For all other situations, just two different values of qoot are used to determine the scaling of the critical Murakami parameter. We show our results graphically in Fig. 3, where we plot $\bar{n}_{e} R / B_{T}$ vs. $1 / q_{c y} \propto I_{p} R / B_{T}$. The primary result is that with flat profiles $\bar{\pi}_{e} R / B_{T}$ depends weakly on $q_{\mathrm{c} x}$, whereas just the opposite is true for the peaked profile runs. We also display in Fig. 3 lines given by the scalings $\overline{n_{e}}=B_{T} / R q_{c y t}$ and $\overline{n_{e}}=2 B_{T} / R q_{c \alpha x}$. Many of the present day estimates for the density limit scaling fall between these two lines (see, for example, Reis. 10 and 11).

As an example of how the electron temperature profite varies with simulation time (i.e., with the Murakami parameter), we show $T_{e}(r, t)$ from simulation $02 \mathrm{~h}$ in Fig. 4. The contraction with incremental increases in the Murakami parameter (due here to decreases in $B_{T}$ and $I_{p}$ ) is clear. The central temperature drops steadily as well. Some variation in the center due to sawtooth oscillations is apparent in Fig. 4, but the profiles remain relatively flat inside of the mixing radius at all times. At $t=8.0 \mathrm{sec}$ in $02 \mathrm{~h}, T_{c}=35$ $\mathrm{eV}$ at the $q=2$ surface $(r \simeq 0.55 \mathrm{~m})$. By the end of the run, the electron temperature at that radius is well below $10 \mathrm{eV}$. The critical parameters of Table I are obtained by interpolating between these two steady states, so that there is no set of profiles corresponding exactly to our disruption criterion. 
In Fig. 5. we plot the toroidal current density as a function of time and radial half-width for simulation 03d. Note that since this run is a density scan, the total plasma current is held fixed at 2.2 M.A. As the temperature profile contracts, so does the current profile. The effect of each incremental addition to the density is evident as the edge of the current profile moves further in. The relatively frequent sawtooth oscillations cause the current density to be more or less lat in the center throughout the simulation. Fote that at the end of this run, the $q=2$ surface is near $r=0.6 \mathrm{~m}$. and the sawtooth mixing radius is approximately $r=0.4 \mathrm{~m}$. Figure 5 clearly shows that virtually all of the current is inside the $q=2$ suriace by the end of the simulation. indicative of an unstable profile. Our disruption criterion is actually met prior to this point. In particular. $T_{e} \simeq 30 \mathrm{el}$ at $t=6.5$ in this run. Again, the ralue of $\overline{\pi_{e}}$ given in Table $I$ is obtained by interpolating between this state and the one found at the end of the simulation.

In Fig. 6, we present. typical electron density profiles from two runs using a titanium impurity. The peaked profile comes from $02 \mathrm{~h}$. and the flat one is taken from 02l. In both cases, the sawtooth mixing radius is $\sim 0.3 \mathrm{~m}$. We should point out that even with the inward pinch, the variations in the density profile during the sawtooth rise are relatively minor. Furthermore. when the titanium impurity is used, the changes in its overall shape as other parameters $\left(I_{p}, B_{T}\right.$, and $\left.\bar{n}_{e}\right)$ are varied are minimal. This is true even for states with severely contracted temperature profiles.

Figure 7 contains a plot of the electron density as a function of time and radial half-width for simulation $06 \mathrm{e}$. This is a flat profile $I_{p}, B_{T}$ scan with carbon impurity: Note that as the contraction proceeds the central density increases to maintain constant $\overline{n_{e}}$. The carbon density profile remains flat thrrughout. The contraction is the result of the reduction in $\langle Z\rangle$ of carbon in the outer zones as the local electron temperature falls with time (see Fig. 2). This effect is not apparent in the titanium runs because of the much lower contribution to $n_{e}$ made by the impurity, $\langle Z\rangle n_{=}$. Note that with $\mathrm{Eq}$. (10), $\langle Z\rangle n_{z} \simeq\left(Z_{\text {eff }}-1\right) /(\langle Z\rangle-1)$. So. there is less electron density due to the impurity in the titanicon suns than in the carbon runs because of the lower $Z_{\text {eff }}$ and the higher $\langle Z\rangle$.

The radiated power per unit volume ii.e., Eq. (9)] as a function of time and radial half-width for simulation $02 \mathrm{~h}$ is displayed in Fig. 8 . The contributions from the primary (low temperature) and secondary peaks of $L\left(T_{e}\right)$ are clear 
(see Fig. 1). Note that even with constant line-averaged elect ron density the magnitude of the primary peak increases as the contraction progresses. This is just due to the higher electron and impurity densities at the smaller radii. One effect that is not clear in Fig. 8 is the change in the radial width of the peaks as the temperature protile steepens. In particular. a steeper (more contracted) temperature profile leads to a narrower peak in the radiated power since the width of $L\left(T_{l}\right)$ in temperature space is fixed. The total radiated power is given by:

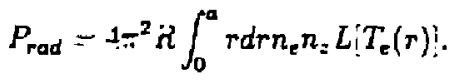

In this expression, a steeper temperature profie leads to a smaller dr for a given $d T_{e}$. Furthermore, because of the contraction, the factor $r$ is smaller as well. Thus, in the contracted state the reductions in the volume element. $r d r$. corresponding to a given peak in $L\left(T_{\varepsilon}\right)$ can counteract to some extent the increased densities, and the total radiated power does not vary so much. These effects are critical to understanding why the maximum Ilurakami parameter for the simulations scales as shown in Fig. 3. We will clarify these points in Sec. IV.

As pointed out in Sec. II, the flat profile runs are obtained by removing the anomalous inward pinch from the particle transport model and setting the edge density close to the volume average (e.g., Fig. 6). This implies that $n_{e} n_{z}$ in the low temperature $\left(T_{e}<100 \mathrm{eV}\right)$ region is much larger than it is for peaked profiles with the same volume-averaged density. Consequently; if all other factors are the same, the total radiated power is greater with flat profiles. In choosing the initial conditions for the flat profile runs, we took this into account. With titanium, we just lowered the impurity concentration relative to that used in the peaked profile cases. In $06 \mathrm{c}$ and $06 \mathrm{e}$, the two carbon runs with flat profiles, we reduced $\overline{n_{e}}$, as indicated in Table I. In Fig. 8 , the radiated power per unit volume increases as the contraction of the temperature profile progresses due to the peaked profiles. With flat profiles: however, the radiated power per unit volume remains approximately constant as the simulation proceeds. This is demonstrated in Fig. 9, taken from 02j. In cases such as this, the total radiated power tends to decrease with increasing Murakami parameter due to the volume element shrinkage noted above.

We feel that these effects are connected directly to the scaling of the critical $\overline{n_{e}} R / B_{T}$ with $q_{c y l}$ noted in Fig. 3 . The crucial point is to realize that 
as $q_{c y l}$ is increased, the $q=2$ surface moves closer to the magnetic axis and. thus. to regions of higher density, assuming a fixed, peaked density profile. At our defined point of disruption, the radiation peaks near the $q=2$ surface. For the higher $q_{\mathrm{cy} l}$ runs, the radiation per unit volume at this point is larger simply because the density is larger. In some sense, this requires a higher ohmic heating power to maintain thermal equilibrium. Since $P_{O H}$ increases with $B_{T} / R$, this requirement translates to a smaller maximum Murakami paraneter.

We should also point out that the initial points for the runs comprising a $q_{c y l}$ scan are, in the 02 series for example, $I_{p}=2.2 \mathrm{MA}$ and $\overline{\pi_{e}}=4.7 \times 10^{19}$

$\mathrm{ml}^{-3}$, with $B_{T}$ computed from $q_{\text {eyl }}$. For the peaked profile runs in this series, it is clear that $P_{O H} / P_{\text {rad }}$ increases with $q_{\mathrm{cy}}$ while $P_{O H}$ remains almost constant. Again, the reason for this is the effect of the density profile shape on $P_{\text {rad. }}$ Hence, our reasoning does not apply just to conditions at disruption.

This analysis can easily be generalized to the case where the volumeaveraged density is allowed to vary for fixed current and toroidal field if the density profile remains fixed. We will quantify these arguments in the next section.

\section{Analytic Calculations}

We now attempt to demonstrate analytically how the scalings noted in Fig. 3 mignt arise. These calculations will be carried out within the confines of our model, the limitations of which have already been noted. Previous theoretical studies have yielded scalings for the maximum Murakami parameter independent of current, ${ }^{17}$ as well as a scaling of the maximum density with current (i.e., a Hugill limit) by the introduction of various physical effects. ${ }^{13,14,16,20}$ Rebut and Green, ${ }^{13}$ for example, relied in part on the scaling of neoclassical transport coefficients to obtain a variation in the Murakami parameter with $q$. Ohyabu ${ }^{24}$ found a $q$ scaling via its effect on the termperature in ohmic equilibrium. The result presented by Campbell et al. ${ }^{20}$ employs an empirical scaling for the radiation that has $g_{\text {oy }}$ dependence in the volume of the radiating layer (and implicitly through the input power). The work presented in Ref. 16 perhaps most closely resembles that discussed in Sec. III. Robert ${ }^{16}$ included the effects of a density gradient when computing the radiation. The contracted current profiles were examined for tea. ng mode stabil- 
ity in order to identify disruptive conditions. As in our Fig. 3. Roberts found that the critical Murakami parameter increased with decreasing $\varphi_{c y l}$. Their calculation lacked, however. self-consistently computed current and density profiles, and did not explicitly include the effects of sawtooth oscillations.

We will show how the presence of a density gradjent can lead to a variation of the Mlurakami parameter with $q_{c y l}$ for these purposes, $q_{c y l}=5 a^{2} B_{T} / R I_{p}$, where $R$ and $a$ (the full simulation minor radius) are in $\mathrm{m}, B_{T}$ is in $\mathrm{T}$, and $I_{\mathrm{p}}$ is in MA. Since the saw tooth oscillations are instrumental in determining the radial transport in these simulations, we find it necessary and even convenient to include their effects here. In general, the line of reasoning used in our calculations is motivated by the detailed results of our simulations. While this limits us to the models assumed therein, it also allows us to check intermediate expressions. insuring a thorough understanding of the phenomena appearing in the simulations.

In order. we will present formulas for the central electron temperature, the radiated power and the ohmic heating power. Then, by equating the radiated power to the ohmic heating power under the corditions assumed to hold at disruption ( $T_{e}=20 \mathrm{eV}$ at $\left.q=2\right)$, a scaling for the critical density can be obtained. Unless otherwise specified, all tinits are MKS, and temperatures are in kel.

We will assume circular fux surfaces and steady state in what follows. Consider first the interior portion of the plasma where the radiated power is regligible. Then, integrating the radial power balance out to the sawtonth mixing radius, we obtain:

$$
\left.r\left(n_{e} \chi_{e} \frac{d T_{e}}{d r}+n_{i} \chi_{i} \frac{d T_{i}}{d r}\right)\right|_{r_{m 11}}=\int_{0}^{r_{m 12}} r d r \text { poH }
$$

where poH $=\eta_{\|} J^{2}$ is the ohmic heating power density. We have applied the boundary condition $d T / d r(r=0)=0$. The parallel resistivity can be writien as

$$
\eta_{\|}=1.65 \times 10^{-9} Z_{e f f} \ln \Lambda_{\gamma_{N C}} T_{e}^{-3 / 2},
$$

with $\ln \lambda$ representing the Coulomb logarithm and $\gamma_{N C}$ the neoclassical resistivity enhancement. ${ }^{38}$

In Eq. (12), the toroidal current density is denoted by $J$. Due to the action of the frequent sawteeth, both $J$ and $T_{e}$ are approximately constant 
over $0 \leq r \leq r_{\operatorname{mix}}$ (see Figs. 4 and 5). In particular, we can use for $d$ its value at the magnetic axis.

$$
J \simeq J_{0}=\frac{2 B_{T}}{\mu_{0} R q_{0}}
$$

Furthermore, we will assume from here on that the safety fectur on axis is unity, $\varphi_{0}=1$.

To evaluate the gradients on the left-hand side of Eq. (12) in general. we need to know the profile shape. With the sawtooth uscillations, we expect a scaling like

$$
\frac{d T_{e}}{d r}\left(r>r_{\text {mix }}\right) \simeq-\left(\frac{T_{e 0}}{a-r_{\text {mix }}}\right)
$$

where $T_{\mathrm{e} 0}$ is the central temperature. We then assume

$$
\frac{d T_{i}}{d r} \simeq \frac{T_{i 0}}{T_{e 0}} \frac{d T_{e}}{d r}
$$

As in our simulations, we use an electron thermal diffusivity

$$
\chi_{e}=\frac{2.6 \times 10^{19}}{n_{e}},
$$

with $\chi_{i}=\chi_{e} / 2$.

Given these assumptions, Eq. (12) yjelds

$$
T_{e 0}=0.76\left[\frac{r_{m i x}\left(a-r_{m i x}\right)}{\left(1+\frac{1}{2} \frac{n i}{n e} \frac{T_{10}}{T_{e 0}}\right)} \bar{\gamma}_{N C} Z_{e / f} \ln \Lambda\right]^{2 / 5}\left(\frac{B_{T}}{R}\right)^{A / 5}
$$

where

$$
\bar{\gamma}_{N C} \equiv \frac{2}{r_{m i x}^{2}} \int_{0}^{r_{m i x}} r d r \gamma_{N C}(r)
$$

accounts for the principal radial variation of the ohmic heating power density out to the mixing radius. Equation (18) does a good job of reproducing the values for the central temperature found in the simulations provided the minor radius, $a$, is taken to be the radius at which $T_{r}=20 \mathrm{eV}$. This is necessary to obtain a reasonable estimate of the temperature gradient found in Eq. (12). Some inaccuracy is allowable here due to the weak dependence of $T_{e 0}$ on $d T_{e} / d r$. 
In general, the total radiated power is given by Eq. (11). Once the plasma is detached, the low temperature peaks of $L\left(T_{e}\right)$ should provide the largest contribution to $P_{\text {rad }}$ (see Fig. 1). This is true for both the low- $Z$ and the high$\mathrm{Z}$ impurities we consider here. So, it is reasonable to approximate $L\left(T_{e}\right)$ by

$$
L\left(T_{\varepsilon}\right)= \begin{cases}L_{p}, & T_{1}<T_{e}<T_{2}, \\ L_{n}, & T_{3}<T_{e}<T_{4}, \\ 0, & \text { otherwise }\end{cases}
$$

where $T_{1}<T_{2}<T_{3}<T_{4}$ and $L_{p}>L_{\text {. }}$. We define corresponding radii, $r_{j} \equiv r\left(T_{j}\right)$ for $j=1$, 4. We include two peaks in Eq. (20) in order :o improve the quantitative accuracy of the resulting expression. With just one peak, however, the fundamental scaling obtained will be essentially the same. In fact. the prucedure could be extended to include an arbitrary number of peaks, but we find that Eq. (20) suffices for present purposes. Looking at the plut in Fig. 1, we estimate $T_{1}=20 \mathrm{eV}, T_{2}=100 \mathrm{eV}, T_{3}=300 \mathrm{eV}, T_{4}=600$ el. $L_{p}=2.5 \times 10^{-31} \mathrm{WVm}^{3}$, and $L,=5.0 \times 10^{-32} \mathrm{WVm}^{3}$ for titanium.

These peaks are relatively narrow in terms of electron temperature. With non-zero temperature gradients, they correspond to small intervals in radius. We can thus reasonably approximate the total radiated power by

$$
\begin{aligned}
P_{\text {rad }} \simeq 2 \pi^{2} R\{ & L_{p}\left(r_{1}-r_{2}\right)\left[r_{1} n_{e}\left(r_{1}\right) n_{3}\left(r_{1}\right)+r_{2} n_{e}\left(r_{2}\right) n_{2}\left(r_{2}\right)\right] \\
& \left.+L_{3}\left(r_{3}-r_{4}\right)\left\{r_{3} n_{e}\left(r_{3}\right) n_{2}\left(r_{3}\right)+r_{4} n_{e}\left(r_{4}\right) n_{2}\left(r_{4}\right)\right]\right\} .
\end{aligned}
$$

The total ohmic input power is given by

$$
P_{O H}=4 \pi^{2} R \int_{0}^{n} r d r \eta_{i l} l^{2}
$$

If the parallel electric field, $E_{\|}=\eta_{\|}{ }^{T}$, is constant over the minot radius, $P_{O H}=(2 \pi R) E_{\|} I_{p}=I_{p} V_{\text {loop, }}$, where $V_{\text {loop }}$ is the loop voltage. We already know $\eta_{\|}$and $J$ at $r=0$. Inserting these expressions into Eq. (22) yields a viable scaling for $P_{O H}$. However, the combination of reoclassical resistivity and our model for the sawtooth oscillations leads to variations in $E_{\|}=\eta_{\| \mid} J$ over $0<r<r_{\text {mix }}$. Namely, if both $J(r)$ and $T_{c}(r)$ flatten in this region following a sawtooth crash, $E_{\|} \propto \gamma_{N C}(r)$. Since the sawtooth period is much less than the resistive skin time, $E_{\|}$remairs peaked off-axis at all times. ${ }^{39}$ On the other hand, for $r>r_{\text {mix }}, E_{\|}$is approximately independent 
of radius in our simulations. Inserting a correction factor to account firr the mriation of $\gamma . v C$ inside the sawtooth mixing radius and using Eq. (13). $F_{\text {oH }} \times(2 \pi R) E_{i_{1}}(r=0) I_{p}$ yields

$$
P_{O H}=0.0165 Z_{e / f} \ln 10.9 \gamma_{N C}\left(r_{m x x}\right)\left(B_{T} I_{p} T_{\mathrm{e0}}^{-3 / 2}\right) .
$$

The factor of 0.9 represents an attempt to calibrate this formula using data from our simulations. The resulting e-pression predicts the ohmic heating power under all conditions to within about $10 \%$ (using the observed $T_{e 0}$ ).

In order to estimate the various radii appearing in Eqs. (18), (21), and (23), we now specialize to the conditions at disruption; namely, $T_{e}=20 \mathrm{eV}$ at the $q=2$ surface. This corresponds to a highly contracted current profile. $J=0$ for $r>r_{\text {mix }}$. But, $J \simeq J_{0}$ for $0<r<r_{\text {mix }}$. Since we know the total current, we can "rite

$$
r_{\mathrm{rntz}}=0.94\left(\frac{\mu_{0}}{2 \pi} \frac{I_{p} R}{B_{T}}\right)^{1 / 2} ;
$$

the factor of 0.94 arises from matching with the simulation results (i.e.. allowing for a slightly broader current profile in reality).

In general, the temperature profile in a contracted state flattens dramatically for $r>r_{1} \equiv r\left(T_{e}=T_{1}\right)$ because of the relatively small amount of heat flow past the primary radiation peak. So, we can reasonably characterize the temperature profile by setting the plasma minor radius $a=r_{3}$. As noted preriously. this a just serves to estimate the temperature gradient in $E_{q}$. (18). For titanium, we take $T_{1}=20 \mathrm{eV}$, so that $r_{t}=r(q=2)=r\left(T_{e}=20 \mathrm{eV}\right)$ when the disruption criterion is satisfied. We suggest that this equality will hold for a general impurity as long as $T_{1} \ll T_{e 0}$. Even if this requires a redefinition of the disruption criterion in order to be consistent (such that $T_{e}=T_{1}$ at $q=2$, for example), the following scalings should still be valid.

Given that the assumption of $J \simeq 0$ for $r>r_{\text {mix }}$ works well in Eq. (24), it should work eren better in calculating $r(q=2)$. In a circular cylinder geometry, the equalities suggested above lead to

$$
a=r_{1}=r(q=2)=\left(\frac{\mu_{0}}{\pi} \frac{I_{p} R}{B_{T}}\right)^{1 / 2} .
$$


To obtain the other radii appearing in Eq. (21), we need to further specify the temperature profile. We will assume

$$
T_{e}(r)= \begin{cases}T_{e 0}, & 0<r<r_{m i x}, \\ \left(T_{e 0}-T_{e a}\right)\left(\frac{a-r}{a-r_{m i x}}\right)^{a T}-T_{e a}, & r_{m i x}<r<a .\end{cases}
$$

Then.

$$
r\left(T_{j}\right)=a-\left(a-r_{\text {mix }}\right)\left(\frac{T_{j}-T_{e a}}{T_{e 0}-T_{e a}}\right)^{1 / a r} .
$$

If we define the edge temperature. $T_{e a}=T_{a}(a)$, to be equal to $T_{1}$ as would follow from the above discussion. this formula holds for $j=1$ as well as $j=2-4$. For simplicity, we will neglect $T_{e a}$ relative to $T_{e 0}$ in using Eq. (2i).

We expect in general that the temperature profile exponent $\alpha_{T}$ will be in the range 1 - 2. Using $d T_{e} / d r$ near the edge (i.e., between $r_{1}$ and $r_{4}$ ) from several simulations to estimate $\alpha_{T}$, we find $\alpha_{T} \sim 1.4$. However, we retain $a_{T}$ in the following expressions for completeness.

Setting $P_{\text {rad }}=P_{O H}$, and making use of Eqs. (18), (24), (25), and (27), we obtain

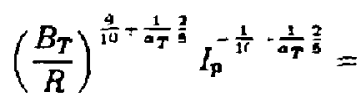

$$
\begin{aligned}
& \frac{44 .}{(0.29)^{1 / \alpha \tau}}\left[\frac{\bar{\gamma}_{N C} / \gamma_{N C}\left(r_{m i x}\right)}{\left(1+\frac{1}{2} \frac{n i}{n e} \frac{T_{10}}{T_{r o}}\right)}\right]^{\frac{3}{5}-\frac{1}{a_{T}} \frac{?}{b}} \\
& \times\left[\gamma_{N C}\left(r_{m i x}\right) Z_{e f \rho} \ln \Lambda\right]^{-\frac{2}{5}\left(\frac{a T-1}{\partial T}\right)}\left\langle L \Delta T r n_{e} n_{F}\right) .
\end{aligned}
$$

where $I_{P}$ is now in MA. We have defined

$$
\begin{aligned}
\left\langle L \Delta T r n_{e} n_{z}\right\rangle \equiv & L_{p}\left(T_{2}-T_{e a}\right)^{1 / a_{T}}\left[r_{1} n_{e}\left(r_{1}\right) n_{x}\left(r_{1}\right)+r_{2} n_{e}\left(r_{2}\right) n_{z}\left(r_{2}\right)\right] \\
+ & L_{4}\left[\left(T_{4}-T_{e q}\right)^{1 / \alpha_{T}}-\left(T_{3}-T_{e a}\right)^{1 / a_{T}}\right] \\
& \times\left[r_{3} n_{e}\left(r_{3}\right) n_{z}\left(r_{3}\right)+r_{4} n_{e}\left(r_{4}\right) n_{z}\left(r_{4}\right)\right]
\end{aligned}
$$

The only thing remaining to do is to determine the scaling of $\left.(L\lrcorner T r n_{e} n_{z}\right)$. We will assume

$$
\left\langle L \Delta T r n_{e} n_{z}\right\rangle \propto \bar{n}_{e}^{-2} \frac{n_{z}}{n_{e}} q_{c y l i}^{p}
$$


we need to estimate the exponent p. The gost scaling comes in not only through the factors $r_{\text {r }}$ through $r_{+}$e.g.. as in Eq. (25). but also through the density profile, as discussed in Sec. III. Nimely, as $q_{r y,}$ is raised. the cuntractiun must proceed to smaller radii in order to satisfy the disruption criterion. At these radii. the electron and impurity densities are higher (for peaked profiles). Hence, the density factors appearing in $\left(L \Delta T r n_{\mathrm{e}} n_{z}\right)$ increase with $q_{c, \mathrm{~s}}$. As pointed out in Sec. III, the overall shape of the profile in the cases with titanium impurity does not vary much when the plasma current and/or the toroidal field are changed. So, for the purposes of estimating $p$, we suppose that the density profiles are fixed.

From Eqs. (24) and (25), we know $r_{m i x}, a \propto q_{c y l}^{-1 / 2} ;$ we assume for simplicity that this scaling is dominant in Eq. (27), $r_{j} \sim q_{c y i}^{-1 / 2}$. Then, we see that with flat profiles (no $q_{c y l}$ scaling for the density factors), $\left.(L\lrcorner T r n_{e} n_{z}\right) x$ ${\overline{n_{e}}}^{2}\left(n_{z} / n_{e}\right) q_{c y l}^{-1 / 2}$.

In the case of peaked profiles, write $n_{e}\left(r_{j}\right) n_{z}\left(r_{j}\right) \times q_{\mathrm{cyl}}^{p^{\prime}}$, then

$$
\frac{d}{d q_{c y l}} n_{\mathrm{e}} n_{x} \times \frac{p^{\prime}}{q_{c y l}} n_{\mathrm{e}} n_{z} .
$$

Taking a profile shape analogous to Eq. (26), with exponents $a_{\mathrm{e}}$ and $\alpha_{z}$ for the electron and impurity density profiles. respectively, we obtain

$$
\left.\frac{d}{d q_{c y l}} n_{e}\left(r_{j}\right) n_{z}\left(r_{j}\right)\right]=-\frac{d s_{j}}{d q_{c y l}}\left(\alpha_{e}+\alpha_{z}\right) \frac{n_{e}\left(r_{j}\right) n_{z}\left(r_{j}\right)}{\left(a_{0}-r_{j}\right)} .
$$

Because there is no contraction of the density profile. we employ $a_{0}$ here, the initial plasma minor radius in the simulations. Using $r_{j} \sim q_{c y i}^{-1 / 2}$ as suggested and referring to Eq. (31), we can determine a local effective exponent,

$$
p^{\prime} \sim \frac{\alpha_{e}+\alpha_{z}}{2} \frac{r_{j}}{a_{0}-r_{j}} .
$$

We expect $\alpha_{e} \sim \alpha_{z} \sim 1$. The scaling is likely to be dominated by that of the radii of the low temperature peaks, i.e., $r_{1}$ and $r_{2}$. Since these never get as small as $a_{0} / 2$ during our simulations, it is reasonable to take $r_{j} /\left(a_{0}-r_{j}\right)>1$; we use $r_{j} /\left(a_{0}-r_{j}\right) \sim 3 / 2$ for clarity. Thus, $p^{\prime} \sim \frac{3}{2}$. With $r_{j} \sim q_{\text {cyl }}^{-1 / 2}$, we finally obtain

$$
\left\langle L \Delta T r n_{e} n_{z}\right\rangle \times \bar{n}_{e}^{2} \frac{n_{z}}{n_{e}} q_{c y l}
$$


for peaked protiles.

Expressing $q_{c y t}$ in terms of $I_{p}$ and $B_{T} / R$. and consjdering just the overall scaling, Eq. (28) yields at fixed $n_{2}, n_{\mathrm{e}}$

$$
\overline{n_{e, \text { crit }}} \propto\left(\frac{B_{T}}{R}\right)^{\frac{9}{50}-\frac{1}{3 a_{T}}-\frac{\varepsilon}{2}} I_{p}^{-\frac{1}{2 N}-\frac{1}{3 v T}-\frac{\varepsilon}{2}} .
$$

Then, inserting $\alpha_{T}=1.4$, and $p=-1 / 2$ for flat profiles, $p=+1$ for peaked profiles,

$$
\overline{n_{e}, \text { Lrit }} \propto \begin{cases}\left(\frac{B_{T}}{R}\right)^{0.84} I_{p}^{-0.16}, & \text { flat profiles, } \\ \left(\frac{B_{T}}{R}\right)^{0.09} I_{p}^{0.59}, & \text { peaked profiles. }\end{cases}
$$

In Tables II and III, we apply this scaling to the results given in Table I. For comparison, we include in these tables the simplest scalings apparent in Fig. 3. For the peaked profile runs. it looks as though something like the familiar Hugill limit, $\tilde{n}_{e} \propto I_{p}$, should apply, while for flat profiles $\overline{n_{e}} \propto B_{T}$ appears to be appropriate.

For the peaked profile cases, Eq. (36) works very well (i.e., the constant of proportionality in Table II does not vary much). Teither of the scalings listed in Table III dues as good a jot of explaining the flat profile data. One of the problems is that the density profiles are not completely flat (see Fig. 6). Furthermore, in the high $q_{e y f}$ cases, the length of the scan is considerable. The number of points along the scan is fixed by code limitations, so that the error in estimating of the critical density is larger for these runs.

The preceding calculations should hold for the runs with carbon impurity as well apart from the estimated $q_{c s l}$ scaling of $\left\langle L \Delta T r n_{e} n_{z}\right\rangle$. Because of the changes in the electron density profile accompanying the temperature profile contraction, it is not clear how this scaling can be determined. On the other hand, the trends apparent in Fig. 3(c) are similar to those found in the simulations with titanium impurity. Namely, with peaked density profiles, $\bar{n}_{e, \text { errit }}$ still scales approximately like the total current; but with flat profiles, the scaling is reduced. We suspect that the effects responsible are similar, but obscured by the contraction of the electron density profile. 


\section{Discussion and Conclusions}

In this section, we comment on how our results relate to experimental observations. But, first, it is appropriate to point out the shortcomings of the present model and to indicate how they impact our results. For instance, the fact that the data of Fig. 3 fall well within the tange of present empirical scalings (for example, ${ }^{11} \bar{n}_{e}<2 B_{T} / R g_{c y}$ ) is somewhat coincidental. This is because we have taken no ste:- to determine self-consistently the impurity concentration in these simulations. In all cases it is specified arbitrarily on input. Since the radiated power is directly proportional to the impurity density, we could extend our results to larger Murakami parameters just by lowering $n_{z} / n_{e}$ as has been noted previously. $9,10,15,17$ The effect is offset somewhat, but not completely by the increase in the ohmic power with $Z_{\text {eJI }}$ as is evident in Eq. (28).

Our model is also inadequate in that coronal equilibrium is known to under-predict the radiation in actual experiments. ${ }^{19}$ By assuming coronal equilibrium, we o:nit effects such as charge exchange recombination of impurities $^{21,40}$ (significant in the presence of high neutral density) and transport of individual impurity charge states on time scales shorter than those over which coronal equilibrium is established. Roberts ${ }^{16}$ points out that including the latter effect leads to smaller critical Murakami parameters, particularly for low $Z$ impurities. Heuristic corrections to the coronal equilibrium model would improve the accuracy of our results to some extent, but would not aid in formulating the general, predictive model that we seek.

Lastly, our disruption criterion is arbitrary. It may not be inadequate, however, especjally for the present scaling arguments. Our guideline is effectively the same as that used by Perkins and Hulse. ${ }^{17}$ They define the density limit as the point at which $P_{\text {rad }}=0.8 P_{O H}$ when evaluated at $q=2$. Since most of the radiation in our runs comes from the low temperature part of the profile, $T_{e}<100 \mathrm{eV}$, we do not satisfy their condition until the temperature profile contracts inside of $q=2$. However, at this point our disruption criterion ( $T_{e}=20 \mathrm{eV}$ at $q=2$ ) is nearly satisfied. Preliminary indications are that use of the stability boundary in $\ell_{i}$ vs. $q$ space given by Cheng et al. ${ }^{37}$ yields nearly equivalent results.

The procedure used by Roberts ${ }^{16}$ represents the next level of sophistication. In Ref. 16, the profiles resulting from an electron energy balance are 
analyzed for tearing mode stability, yielding saturated island widths. The disruption mechanism discussed there most closely resembling the criterion we employ is due to the interaction between the $2 / 1$ tearing mode and the cold plasma outside the radiating boundary. It appears that the widths of the islands in their work do not change greatly as conditions are varied. If this really is the case, then improving our disruption criterion involves only redefining the surface $(q>2)$ that must be reached by the cold piasma. The basic scalings arrived at above should still hold. The tearing mode package being installed in BALDCR ${ }^{22}$ will be able to not only calculate saturated island widths, but will also treat self-consistently the effects of localized current profile flattening.

For the purposes of identifying the basic scaling of the density limit in ohmic discharges, our model should be adequate. In particular. the effects of density gradient and sawteeth we treat here do lead to interesting results that are not likely to be affected by the above-mentioned shortcomings.

We now compare our results with some specific experimental observations. First. we consider the density limit formula presented in Ref. 20:

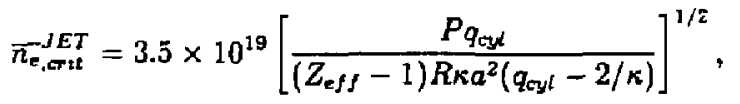

where $P$ is the total input power in $\mathrm{MW}$, and $\kappa$ is the plasma elongation $(\kappa \simeq 1$ for a TFTR geometry). This relation is obtained by equating an empirical scaling for the radiated power with the input power. Equation (37) applies only if $Z_{e f f}$ is not close to one and if light impurities (e.g., oxygen and carbon) dominate the radiated power. All of our runs with carbon impurity fall into this category. Inserting $P=P_{O H}$ and $Z_{\text {eff }}$ for a particular time during each simulation, we find that on the average $\overline{n_{e}} / \overline{n_{e}, c r i t} \sim 2$, where $\overline{n_{e}}$ is taken as in Table I. Because of the variations in $P_{O H}$ and $Z_{e f f}$ during our runs, $\bar{n}_{e, c r s:}^{J E T}$ cannot be uniquely determined. It is fairly clear that $\bar{n}_{e, c r i t}^{J E T}$ does not exhibit the same current scaling as displayed in Fig. 3(c). Namely, for each pair of runs with carbon impurity (05b and $05 \mathrm{c}$, for example), the $\overline{n_{e}}$ at the two different values of $q_{\mathrm{cyl}}$ in Table $\mathrm{I}$ is either similar, or defined to be the same ( $I_{p}, B_{T}$ scans). Yet, their formula predicts a noticeable variation with $q_{c y t}$ in all cases, as is clear from Eq. (37). The source of this discrepancy is uncertain.

The fact that the critical densities predicted by our model are about a 
factor of two below those found with $\mathrm{Eq}$. (37) indicates that the magnitude of the radiation obtained using coronal equilibrium is approximately four times smaller than that observed experimentally e.g., see Eq. (28). This is consistent with the calculation presented in Ref. 19, where the coronal equilibrium radiation is a factor of five to ten smaller than that computed from the radiation data.

In gas-fuelled, ohmic discharges, TFTR has obtained Murakami parameters up to ${ }^{23} \overrightarrow{n_{e}} R / B_{T}=3.2 \times 10^{19}$. In present TFTR shots, the dominant impurities are carbon and oxygen. From Table $I$, we see that the maximum value in our runs is $\overline{n_{e}} R / B_{T}=5.66 \times 10^{\text {t9 }}$ (from 06a). This discrepancy is again consistent with our underestimatirg the radiation by about a factor of four. On the other hand, pellet injection into TFTR yields much higher densities, $\overline{n_{e}}=1.4 \times 10^{20}$ and $\overline{n_{e}} R / B_{T}=6.5 \times 10^{19}$. This indicates the importance of fuelling and recycling effects; ${ }^{23}$ they will be the focus of future investigations. ${ }^{31}$

The detached plasmas studied in $\operatorname{TFTR}^{32,33}$ are very similar to the steadystate contracted plasmas investigated here. Because these TFTR discharges remain in the detached state for many energy confinement times without disrupting, they serve as a source of experimental data with which we can compare and calibrate our model. For now we would just like to point out some of the similarities between the simulations presented here ard the experiments discussed in Refs. 32 and 33 . Much as we have done, the detached plasmas in TFTR are produced by ramping down the current at constant line-integrated density. It is convenient for us to also ramp down the toroidal field in order to maintain a constant $q_{\text {cyl }}$.

Experimentally, the resulting detached plasma has a contracted temperz.ture profle ( $T_{e}<50 \mathrm{eV}$ in the cold edge region), bounded by a radiating layer. The electron density profile also appears to be somewhat contracted. ${ }^{33}$ The power balance calculations reported by Strachan et al. ${ }^{33}$ indicate that all of the ohmic power appears as radiated power; that is, there is little or no heat conducted or convected to the limiter. All of these features are consistent with our simulations employing a carbon impurity.

There are several other trends mentioned in Ref. 33 that are reminiscent of behaviors found in our simulations. First, a decrease in the effective minor radius (defined via the temperature profile) can be brought about by either increasing the central electron density or decreasing the plasma current. An 
experimental scaling for this has been determined:

$$
a \times l_{p}^{0.64 \pm 0.05} n_{e}(0)^{-0.3=0.04} \text {. }
$$

The data with carbon impurity we have presented are n'st extensive enough to allow a detailed comparison with this expression, but preliminary indications are that our simple model yields a weaker current scaling than this.

Secondly, the total radia' : puwn und the a diated power per unit volusu increase with central electron density both in the experiments and in our simulations. We cannot say anything definite about how these quantities vary with plasma current in our runs because of the increases in central density that are required during the profile contraction to maintain constant line-averaged density (see Fig. 7 ) in the $I_{p}, B_{T}$ scans. We do note that in run $06 e$, the total radiated power decreases with plasma current, despite an increase in $n_{e}(0)$ (see Fig. 7). In Ref. 33 it is noted that the central electron temperature falls as the central density is increased. This is also the case for our two density scans with carbon impurity ( $05 \mathrm{~b}$ and $v i s$ ).

Finally, Strachan et al. ${ }^{33}$ point out that the safety factor at the effective edge of the plasma decreases with increasing $n_{e}(0)$, down to about $q=2$. Further attempts to increase the central density lead to disruption. This is another indication that our disruption c "ecion, $T_{e}=20 \mathrm{eV}$ at $q=2$, is reasonable. Future work will attempt to make more quantitative comparisons between the simulations and experiments.

In summary, we have developed a relatively simple transport model that yields qualitatively correct scalings for the density limit in ohmic tokamak discharges. The emphasis here has been on effects of the density profile. In particular, we have pointed out that at constant line-averaged electron density, a flat profile generates more radiated power than a peaked profile just due to the higher edge density.

The profile shape also impacts the current scaling of the critical electron density. Namely, with peaked electron density profiles, the location of the $q=2$ surface moves inward to regions of higher density as $q_{\mathrm{cyd}}$ is raised. This leads to an effectively higher radiated power than at smaller $q_{c \neq}$ in the maximally contracted state where the peak of the radiation is near $q=2$. This is also true of the initial, uncontracted states used in our simulations. The end result is a decrease in the allowable Murakami parameter as $q_{\mathrm{cy}}$ is increased. Of course, the argument does not hold for flat profiles, and very 
little scaling of the Murakami parameter with $q_{c y k}$ is noted in those cases. These trends are clear in Fig. 3 and have been confirmed by our analytic calculations.

These two density profile effects suggest that if all other factors (impurity concentration, average density, etc.) are the same, discharges with peaked profiles will yield larger critical Murakami parameters than those with flat profiles. So, in an actual experimental data base consisting of a large number of shots produced under various conditions, the ones yiejding the highest Murakami parameter at a given $q_{\mathrm{cyl}}$ will be the ones with the most peaked profiles.

It has been observed experimentally that $Z_{\text {eff }}$ decreases with increasing density. ${ }^{23}$ We have not taken this into account in our simulations (we keep $Z_{\text {eff }}$ approximately constant as the density is varied). It would act to enhance the $q_{c y l}$ scaling of the maximum Murakami parameter shown in Fig. 3. For example, if we write instead of $\mathrm{Eq}$. (30)

$$
\left\langle L \Delta T r n_{e} n_{z}\right\rangle \propto \overline{n_{e}} n_{z} q_{c y l}^{p}
$$

and hold $n_{x}$ constant, the exponents in Eqs. (35) and (36) double. Then, the scaling of $\overline{n_{e}, r i t}$ with current is stronger, but still close to linear. In this way, our relatively simple model can account for the overall shape of the Hugill diagram.

The effects of pellet injection and auxiliary heating will be examined in the near future. 32 The importance of profile shape indicates that the central fuelling that can be provided by pellet injection allows higher line-averaged densities to be attained. This result is clear in present experiments. ${ }^{10,11,23}$ Peaked profiles are of even greater importance in a reactor due to their impact on the fusion power output., ${ }^{1,3}$ It is also known that auxiliary heating can increase the maximum allowable Murakami parameter. ${ }^{9-11}$ Clearly, our model will yield this result since $\mathrm{jt}$ is based upon a radiative power balance. Greenwald ${ }^{9,10}$ has proposed that mechanisms insensitive to input power and impurity concentration may actually be responsible for the current scaling of the density limit. Future work will attempt to clarify this picture.

Our next step in developing a predictive model for the density limit will be improving the radiation model in the BALDUR code, allowing it to treat efiects such as charge exchange recombination ${ }^{40}$ and transport of impurity charge states. ${ }^{21}$ Attempts will also be made to treat the scrape-off layer and 
boundary conditions in a more realistic fashion (see. for example, Ref. 41 ). These steps will be undertaken with guidance from experimental data.

\section{Acknowledgments}

The author wishes to acknowledge fruitfu] djscussions with Drs. G. Bateman, D. Post, C. Singer, F. Perkins, P. Hulse, and M. Greenwald. This work was supported by U.S. DoE Contract No. DE-AC02-76-CHO-3073. 


\section{References}

'D. P. Stotler. D. Post, and G. Bateman, Princeton Plasma Physics Laboratory Report No. PPPL-2463 (1987); Fusion Technol. (in press).

${ }^{2}$ D. Fost, WV. Houlberg, G. Bateman, L. Bromberg, D. Cohn, P. Colestock, M. Hughes, D. Ignat, R. Izzo, S. Jardin, C. Kieras-Phillips, L. P. Ku, G. Kuo-Petravic, B. Lipschultz, R. Parker, C. Paulson, Y'-K. M. Peng, II. Petravic, M. Phillips, N. Pomphrey, J. Schmidt, D. Strickjer, A. Todd, N. L̈ckan, R. White, S. Wolfe, and K. Young, Physica Scripta T16. 89 (198i).

${ }^{3}$ D. P. Stotler and Glenת Bateman, Princeton Plasma Physics Laboratory Report No. PPPL-2510 (1988); Fusion Techrol. (to be published).

'S. .I. Kaye and R. J. Goldston, Nucl. Fusion 25, 65 (1985).

${ }^{5}$ C. Singer, D. Post, D. Mikkelsen, M. Redi, A. McKenney, A. Silverman, F. Seidl, P. Rutherford, R. Hawryluk, W. Langer, L. Foote, D. Heifetz, W. Houlberg, M. Hughes, R. Jensen, G. Lister, and J. Ogden, Princeton Plasma Physics Laboratory Report No. PPPL-2073 (1986); Comput. Physics Commun. (in press).

${ }^{6} \mathrm{G}$. Bateman, in Proceedings of the 1985 Trieste Spring College on Plasma Physics (to be published).

'M. Murakami, J. D. Callen, and L. A. Berry, Nucl. Fusion 16, 347 (19i6).

'K. B. Axon, W. H. M. Clark, J. G. Cordey, M. Cox, S. J. Fielding. R. D. Gill, J. Hugill, P. Lomas, J. W. M. Paul, B. A. Powell, R. Prentice, D. F. Start, D. D. R. Summers, and P. Thomas, in Plasma Physics and Controlled Nuclear Fusion Research 1980, Proceedings of the 8th Internatiolal Conference, Brussels, (IAEA, Vienna, 1981), Vol. 1, p. 413.

${ }^{9}$ M. Greenwald, M. Besen, F. Camacho, C. Fiore, M. Foord, R. Gandy, C. Gomez, R. Granetz, D. Gwinn, S. Knowiton, B. LaBombard, B. Lipschultz, H. Manning, E. Marmar, S. McCool, J. Parker, R. Parker, R. Petrasso, P. Pribyl, J. Rice, D. Sigmạr, Y. Takase, J. Terry, R. Waterson, and S. Wolfe, in Plasma Physics and Controlled Nuclear Fusion Research 
1986, Proceedings of the 11th International Conference, Kyoto (1.AEA. Vienna, 1987), Vol. 1, p. 139.

${ }^{10}$ M. Greenwald, J. Terry, S. Wolfe, S. Ejima. M. Bell, S. Kaye, and G. H. Neilson, MIT Plasma Fusion Center Report No. PFC/JA-86-22 (1988).

"JET Team, in Plasma Physics and Controlled Nuclear Fusion Research 1986, Proceedings of the 11 th International Conference, Kyoto (IAEA, Vienna, 1987), Vol. 1, p. 31 .

12. Gibson, Nucl. Fusion 16, 546 (1976).

${ }^{13} \mathrm{P}$. H. Rebut and B. J. Green, in Plasma Physics and Controlled Nuclear Fusion Research 1976, Proceedings of the 6th International Conference, Berchtesgaden (IAEA, Vienna, 197T), Vol. 2, p. 3.

14. Ohyabu, Nucl. Fusion 19, 1491 (1979).

${ }^{15}$ D. E. T. F. Ashby and M. H. Hughes, Nucl. Fusion 21, 91 (1981).

${ }^{16}$ D. E. Roberts, Nucl. Fusion 23, 311 (1983).

${ }^{17}$ F. W. Perkins and R. A. Hulse, Phys. Fluids 28, 1837 (1985).

${ }^{18}$ D. E. Post, R. V. Jensen, C. B. Tarter, W. H. Grassberger, and W. A. Lokke, At. Data Nucl. Data Tables 20, 397 (1977).

${ }^{19}$ K. Behringer, A. Boileau, F. Bombarda, G. B. Denne, W. Engelhardt, M. J. Forrest, G. Fussmann, R. Giannella, N. A. Gottardi, M. Von Hellermann, L. Horton, H. JäckeI, C. Jupén, E. Källne, K. D. Lawson, G. Magyar, G. M. McCracken, P. D. Morgan, E. R. Müller, N. J. Peaçock, J. Ramette, B. Saoutic, M. F. Stamp, H. P. Summers, G. Tallents, and A. Weller, in Plasmo Physics and Contralled Nuclear Fusion Research 1986, Proceedings of the I1th International Conference, Kyoto (IAEA, Vienna, 1987), Vol. 1, p. 197.

${ }^{20}$ D. J. Campbell, P. A. Duperrex, A. W. Edwards, R. D. Gill, C. W. Gowers, R. S. Granetz, M. Hugon, N. Lopes Cardozo, M. Malacarne, D. C. Robinson, F. C. Schüller, P. Smeulders, J. A. Snipes, P. E. Stott, G. Tonetti, B. J. D. Tubbing, A. Weller, and J. A. Wesson, in Plasma Physics and 
Controlled Nuclear Fusion Research 1986, Proceedings of the 11 th International Conference, Kyoto (IAEA, V'ienna. 1987), Vol. 1. p. 433.

${ }^{21}$ K. A. Hulse, Nucl. Technol./Fusion 3, 259 (1983),

${ }^{22}$ Glenn Bateman, Bull. Am. Phys. Soc. 32, 191 i (1987).

${ }^{23}$ R. J. Hawryluk, V. Arunasalam, J. D. Bell, M. G. Bell, M. Bitter, et al., Princeton Plasma Physics Laboratory Report No. PPPL-2390 (1986).

${ }^{24}$ INTOR Group, International Tokamak Reactor, Phase Two A, Part II, Report of the International Tokamak Reactor Workshop, 1984 \& 1985 , (IAEA, Vienna, 1986).

${ }^{25}$ MI. Greenwald, D. Gwinn, S. Milora, J. Parker, R. Parker, S. Wolfe, M. Besen, B. Blackwell, F. Camacho, S. Fairfax, C. Fiore, M. Foord, R. Gandy, C. Gomez, R. Granetz, B. LaBombard, B. Lipschultz, B. Lloyd, E. Marmar, S. McCool, D. Pappas, R. Petrasso, M. Porkolab, P. Pribyl, J. Rice, D. Schuresko, Y. Takase, J. Terry, and R. Watterson, in Plasma Physics and Controlled Nuclear Fusion Research 1984, Proceedings of the 10th International Conference, London (IAEA, Vienna, 1985), Vol. 1, p. 45.

${ }^{26}$ C. S. Chang and F. L. Hinton, Phys. Fluids 25, 1493 (1982).

${ }^{27}$ M. H. Redi, W. M. Tang, P. C. Efthimion, D. R. Mikkelsen, and G. L. Schmidt, Nucl. Fusion 27, 2001 (1988).

${ }^{28}$ C. B. Tarter, J. Spectros. Radia. Trans. 10, 531 (19i7).

${ }^{29} \mathrm{G}$. Bateman, Princeton Plasma Physics Laboratory Report No. PPPL-2373 (1986); Fusion Technol. (in press).

${ }^{30}$ B. B. Kadomtsev, Sov. J. Plasma Phys. 1, 389 (1975). B 13, 3895 (1980).

${ }^{31}$ D. P. Stotier and Glenn Bateman, in Proceedings of the Sherwood Theory Meeting, Gatlinburg, Tennessee, 1988 (Oak Ridge National Labotatory, Oak Ridge, 1988), Paper 3D37. 
32 J. D. Strachan, F. P. Boody, C. Bush, B. Grek. R. J. Hawryluk, D. Heifetz. H. W. Iendel, K. W. Hill, D. W. Johnson. A. T. Ramsey. S. Sesnic, J. F. Schivell, .1. Shimada. G. Taylor, and M. C. Zarnstorff. in Proceedings of the European Conf. on Plasma Physics and Controlled Fusion (European Physical Suciety, Budapest, Hungary. 1985), Part I, p. 339.

${ }^{33}$ J. D. Strachan. F. P. Boody, C. E. Bush, S. A. Cohen, B. Grek, L. Grisham, F. C. Jobes, D. W. Johnson, D. K. Mansfield, S. S. Medley, W. Morris, H. K. Park, J. Schivell, G. Taylor, K. L. Wong, S. Yoshikawa, M. C. Zarnstorff, and S. J. Zweben, J. Nucl. Mater. 145 - 147. I86 (1987).

${ }^{34}$ John Wesson, Tokamaks (Clarendon Press, New York. 198T).

${ }^{35}$ A. Syckes and J. A. Wesson, Phys. Rev. Lett. 44. 18 (1980).

${ }^{36}$ D. Bartlett, D. Campbell, A. Custley, P. A. Duperrex, A. Edwards, R Gill, N. Gottardi, R. Granetz, P. Haynes, T. Hender, M. Hugon, H. Jäckel, E. Lazzaro, N. Lopes Carduzo, M. Lurentz Guttardi. T. Oyevaar, X. Saimon, F. Schüller, P. Smeulders, J. Snipes, P. Stott, G. Tonetti, D. Ward, A. Weller, and J. Wesson. Bull. Am. Phỵs. Soc. 32, 1838 (1987).

${ }^{37}$ C. Z. Cheng, H. P. Furth, and A. H. Buozer. Plasma Phys. and Controlled Fusion 20, 351 (1987).

${ }^{38}$ S. P. Hirshman and D. J. Sigmar, vنucl. Fusion 21, 1079 (1981).

${ }^{39}$ F. Alladio and G. Vlad, Phys. Fluids 31, 602 (1988).

${ }^{40}$ R. A. Hulse, D. E. Post, and D. R. Mikkelsen, J. Phys. B 13, 3895 (1980).

"J. M. Ogden, C. E. Singer, D. E. Post, R. V. Jensen, and F. G. P. Seidl, IEEE Trans. Plasma Sci. PS-9, 274 (1981). 


\begin{tabular}{|c|c|c|c|c|c|c|c|c|}
\hline run & $\bar{Z}$ & scan & profile & $1 q_{c y l}$ & $\overline{n_{e, 19}}$ & $I_{p}$ & $B_{T}$ & $\overline{n_{e .19} R: B_{T}}$ \\
\hline $02 d$ & 22 & $I_{p}, B_{T}$ & peaked & 2.8 & 4.70 & 1.50 & 3.20 & 3.75 \\
\hline $02 f$ & 22 & $I_{p}, B_{T}$ & peaked & 3.4 & 4.70 & 1.42 & 3.71 & 3.26 \\
\hline $02 \mathrm{~g}$ & 22 & $I_{p}, B_{T}$ & peaked & ; 3.9 & 1.70 & 1.41 & 4.22 & 2.86 \\
\hline $02 \mathrm{~h}$ & 22 & $I_{p}, B_{T}$ & peaked & 4.4 & 4.70 & 1.29 & $\$ .38$ & 2.76 \\
\hline $02 i$ & 22 & $I_{p}, B_{T}$ & flat & 2.8 & 4.70 & 1.13 & | 2.41 & $5.0 !$ \\
\hline $02 j$ & 22 & $I_{\mathrm{p}}, B_{\mathrm{T}}$ & flat & 3.4 & 4.70 & 0.82 & 2.48 & 4.87 \\
\hline $02 \mathrm{k}$ & 22 & $I_{p,} B_{T}$ & flat & 3.9 & 4.70 & 0.92 & 2.40 & 5.03 \\
\hline $02 !$ & 22 & $I_{p}, B_{T}$ & flat & 4.4 & 4.70 & 0.78 & 2.62 & 4.61 \\
\hline $03 c$ & 22 & $\overline{n_{e}}$ & peaked & 2.8 & $\begin{array}{l}1 \quad 6.30 \\
\end{array}$ & 2.20 & 4.70 & 3.44 \\
\hline 03d & 22 & $\overline{n_{e}}$ & peaked & 3.9 & 6.60 & 2.20 & 6.60 & $2.5 \bar{i}$ \\
\hline $05 b$ & 6 & $\overline{n_{e}}$ & peaked & 2.8 & 10.0 & 2.20 & $: \quad 4.70$ & 5.47 \\
\hline $05 c$ & 6 & $\overline{n_{e}}$ & peaked & 3.9 & 9.90 & 2.20 & 6.60 & 3.86 \\
\hline $06 a$ & 6 & $J_{p}, B_{T}$ & peaked & 2.8 & 8.00 & 1.70 & $\quad 3.63$ & 5.66 \\
\hline $06 \mathrm{~b}$ & 6 & $I_{p}, B_{T}$ & peaked & 3.9 & 8.00 & 1.66 & i 4.99 & 4.12 \\
\hline $06 \mathrm{c}$ & 6 & $\dot{I}_{p}, B_{T}$ & flat & 2.8 & 6.00 & 1.75 & $: \quad 3.74$ & 4.12 \\
\hline $06 e$ & 6 & $I_{p}, B_{T}$ & flat & 3.9 & 6.00 & 1.47 & 4.40 & 3.50 \\
\hline
\end{tabular}

Table I: Simulation parameters; $Z$ indicates impurity atomic number. Flat and peaked describe the density profile. Units are $\bar{n}_{\mathrm{e}, 19}: 10^{1 \theta} \mathrm{m}^{-3} . B_{T}: \mathrm{T}$. $I_{p}: M A$, and $R=2.57 \mathrm{~m}$.

\begin{tabular}{|c|c|c|}
\hline run & $\bar{n}_{e, 19} /\left(B_{T}^{0.09} J_{p}^{0.59}\right)$ & $\overline{n_{e, 19} / I_{p}}$ \\
\hline $02 \mathrm{~d}$ & 3.33 & 3.13 \\
$02 \mathrm{f}$ & 3.41 & 3.31 \\
$02 \mathrm{~g}$ & 3.38 & 3.33 \\
$02 \mathrm{~h}$ & 3.53 & 3.64 \\
$03 \mathrm{c}$ & 3.43 & 2.86 \\
$03 \mathrm{~d}$ & 3.51 & 3.30 \\
\hline
\end{tabular}

Table II: Constant required in Eq. (36) and in $\overline{n_{e}} \propto I_{p}$ by data in Table I. 


\begin{tabular}{cc:c}
\hline run & $\bar{n}_{e, 19} /\left(\bar{B}_{T}^{084} I_{p}^{-0.16}\right)$ & $\bar{n}_{e .19} / \bar{B}_{T}$ \\
\hline $02 \mathrm{i}$ & 2.29 & 1.95 \\
$02 j$ & 2.13 & 1.90 \\
$02 \mathrm{k}$ & 2.23 & 1.96 \\
$02 \mathrm{j}$ & 2.01 & 1.79 \\
\hline
\end{tabular}

Table III: Constant required in Eq. (36) and in $\overline{n_{e}} \times B_{T}$ by data in Table I. 


\section{Figures}

FIG. 1. Dependence of the radiated power per unit whlume. divided by the impurity and electron densities on electron temperature. for (a) titanium and (b) carbun computed from results of Refs. 18 and 28. respectively.

FIG. 2. Dependence of the average charge in coronal equilibrium on electron temperature for (a) titanium and (b) carbon computed from results of Refs. 18 and 28, respectively.

FIG. 3. Plot of the data from Table I in Hugill space. Lines for $\overline{n_{e}}=$ $B_{T} / R g_{\mathrm{cy} /}$ and $\overline{n_{\mathrm{e}}}=2 B_{T} / R q_{c y l}$ are included for comparison. In (a) and (b) we show data from runs with titanium impurity; in (c), carbon was used. Open (closed) markers indicate peaked (flat) density profiles. Also $I_{p}, B_{T}$ scans are denoted by square markers, and $\overline{n_{e}}$ scans by circular markers.

FIG. 4. Election temperature as a function of time and radial half-width for simulation $02 \mathrm{~h}$.

FIG. 5. Toroidal current density as a function of simulation time and radial half-wideh for run 03d.

FIG. 6. Electron density as a function of radial half-width for $02 \mathrm{~h}$ and $02 \mathrm{l}$. In both cases. the profiles were taken at the end of the initial (uncontracted) steady state.

FIG. 7 . Electron density as a function of radial half-width and time for simulation $06 \mathrm{e}$.

FIG. 8. Radiated power per unit volume given by Eq. (9) as a function of time and radial half-width for simulation $02 \mathrm{~h}$.

FIG. 9. Radiated power per unit volume given by Eq. (9) as a function of time and radjal half-width for simulation $02 \mathrm{j}$. 

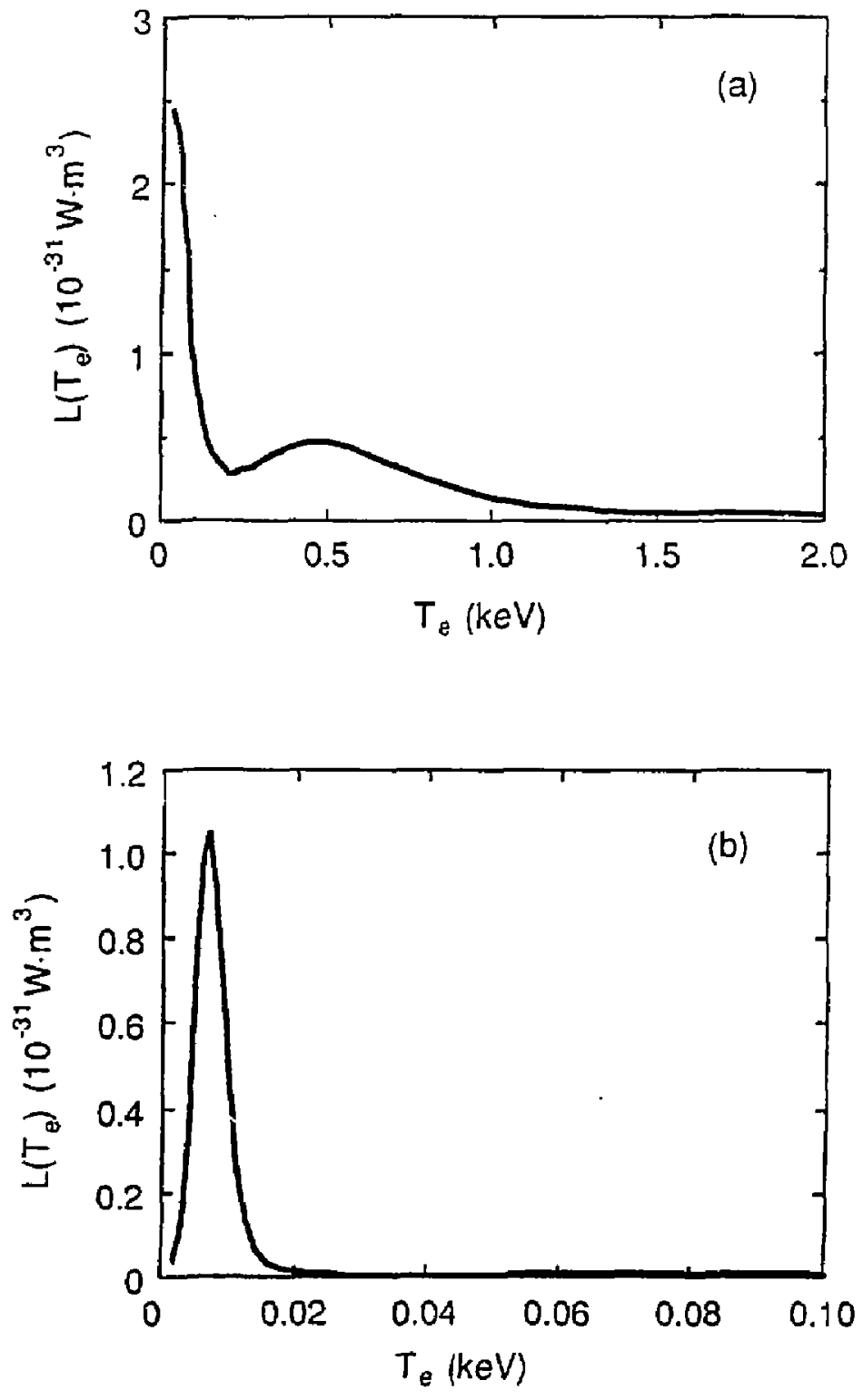

FIG. 1 

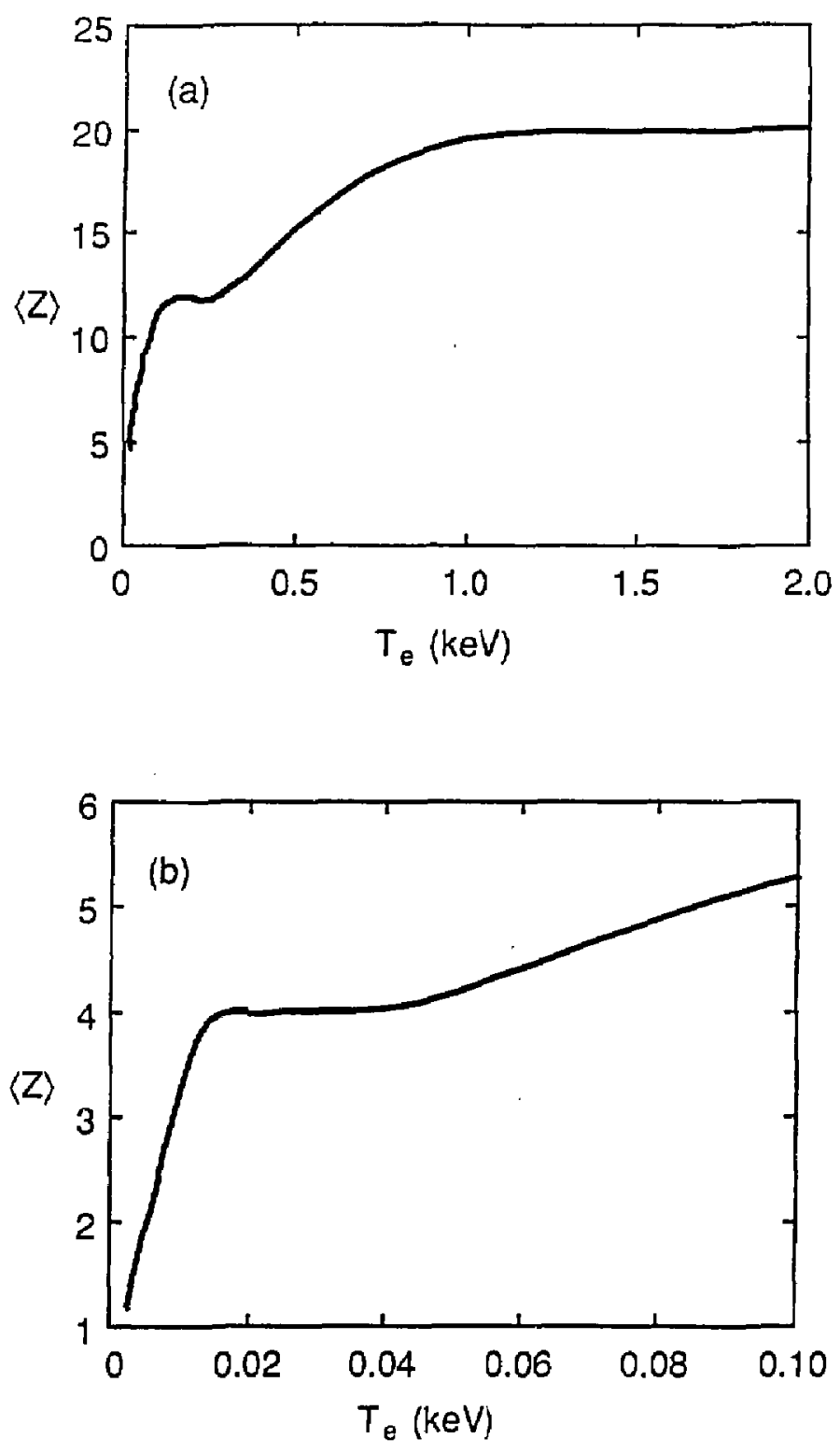

FIG. 2 

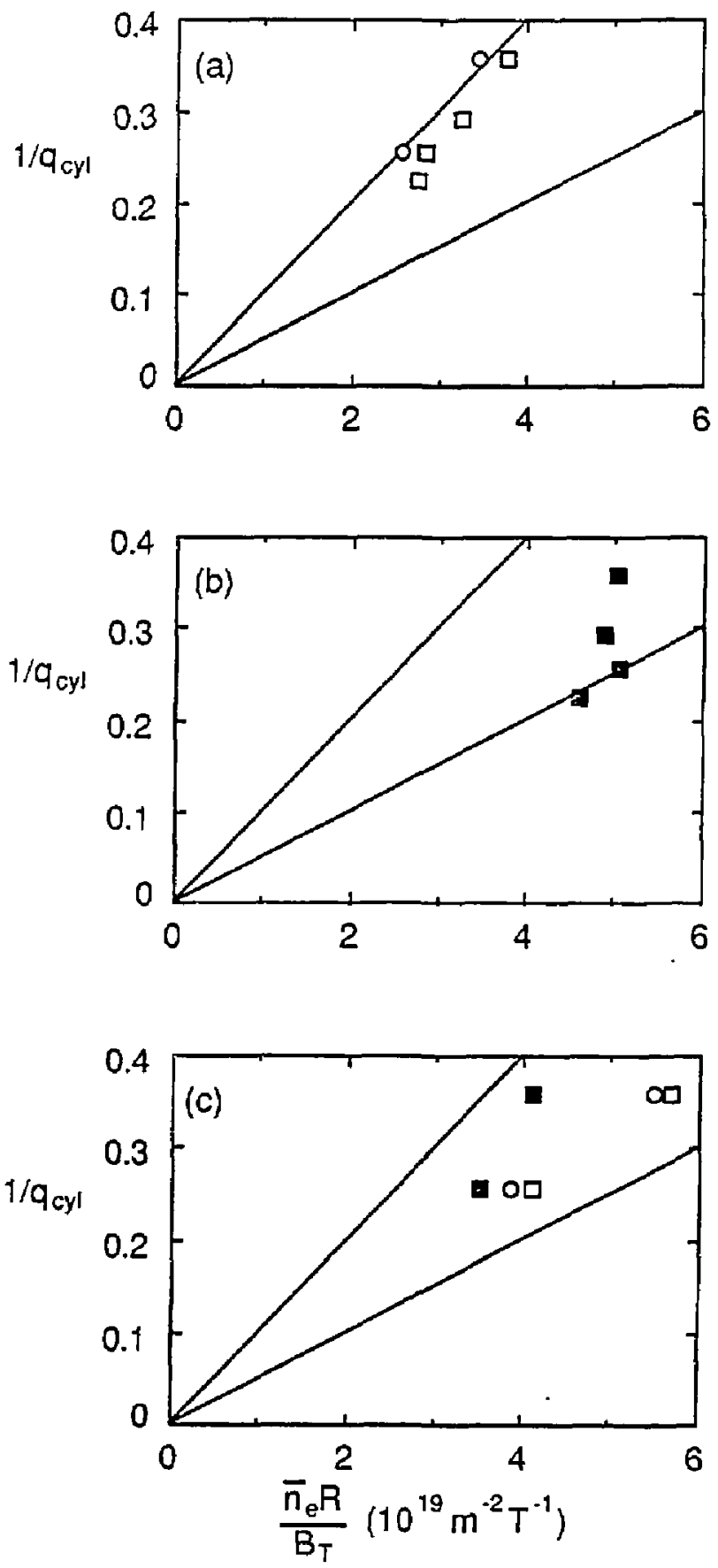

FIG. 3 


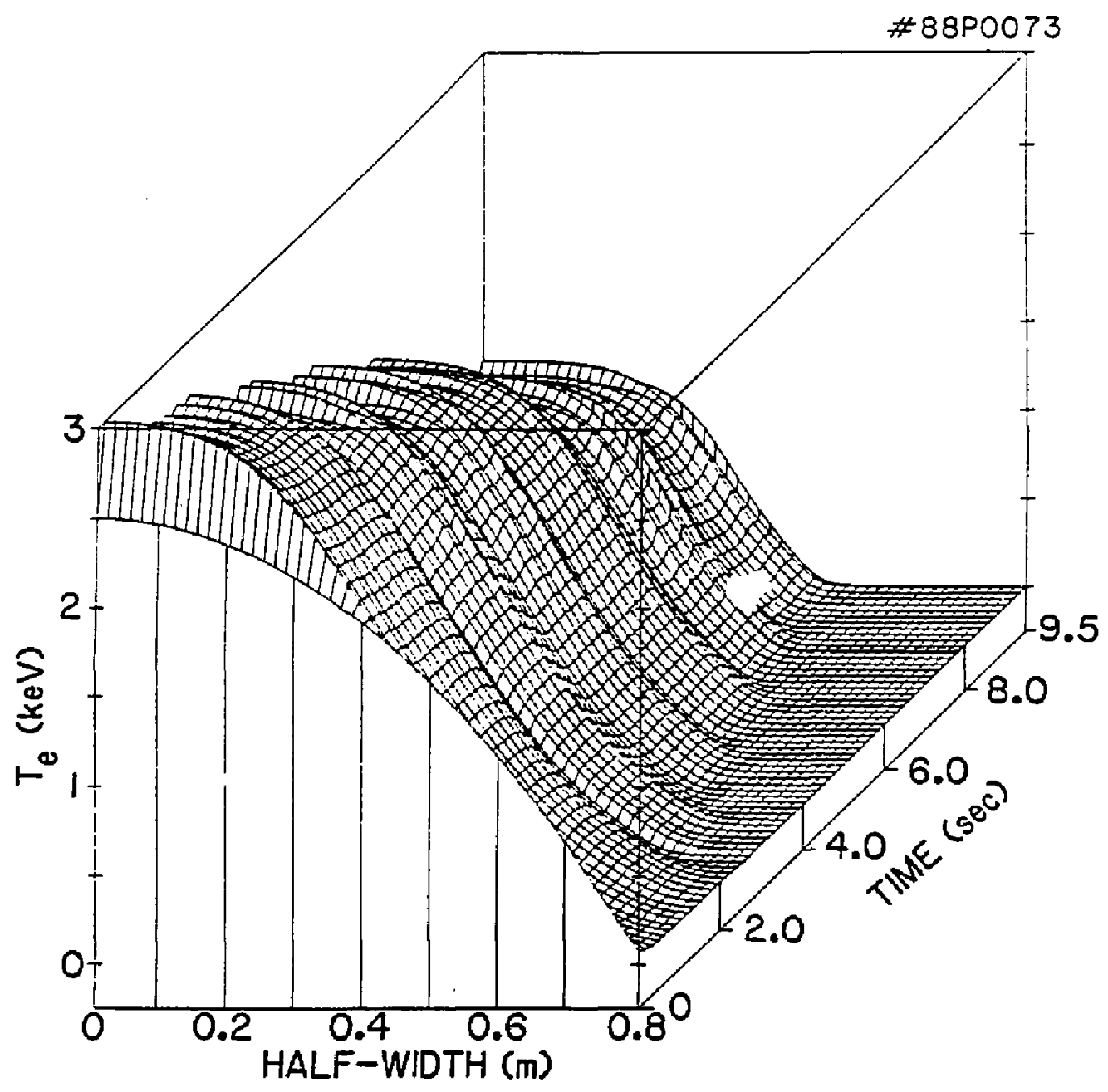

FIG. 4 


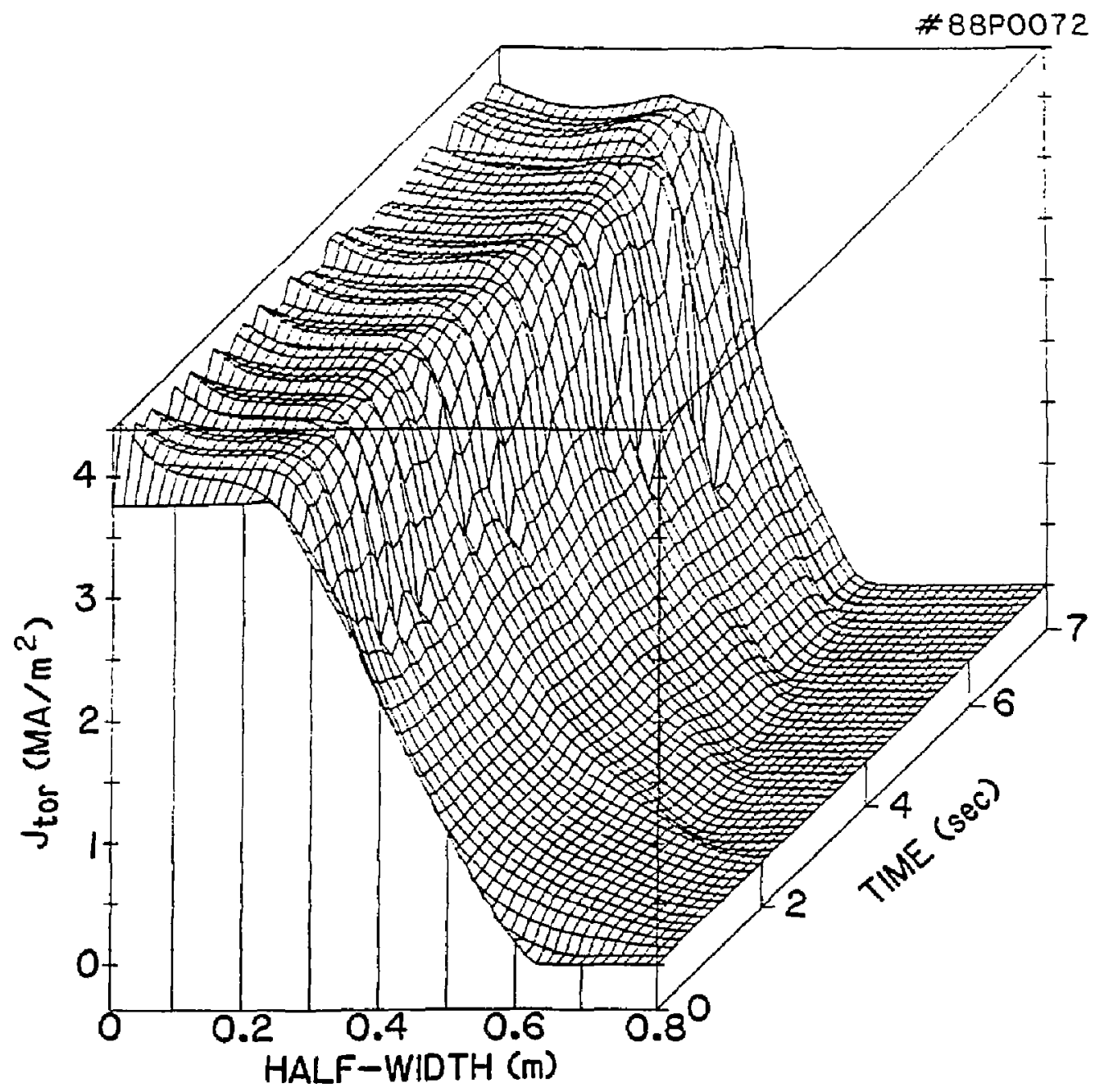

FIG, 5 


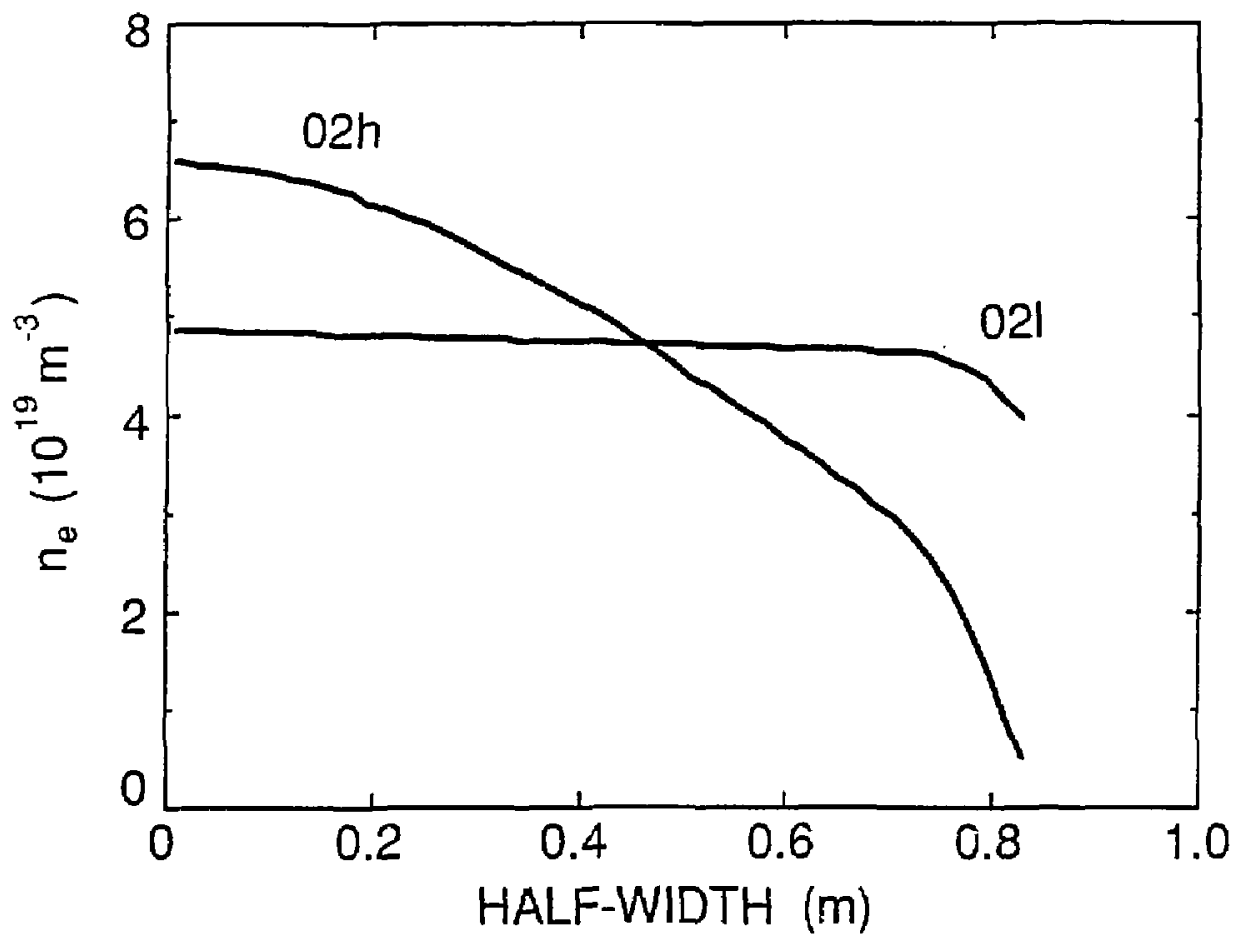

EIG. 6 


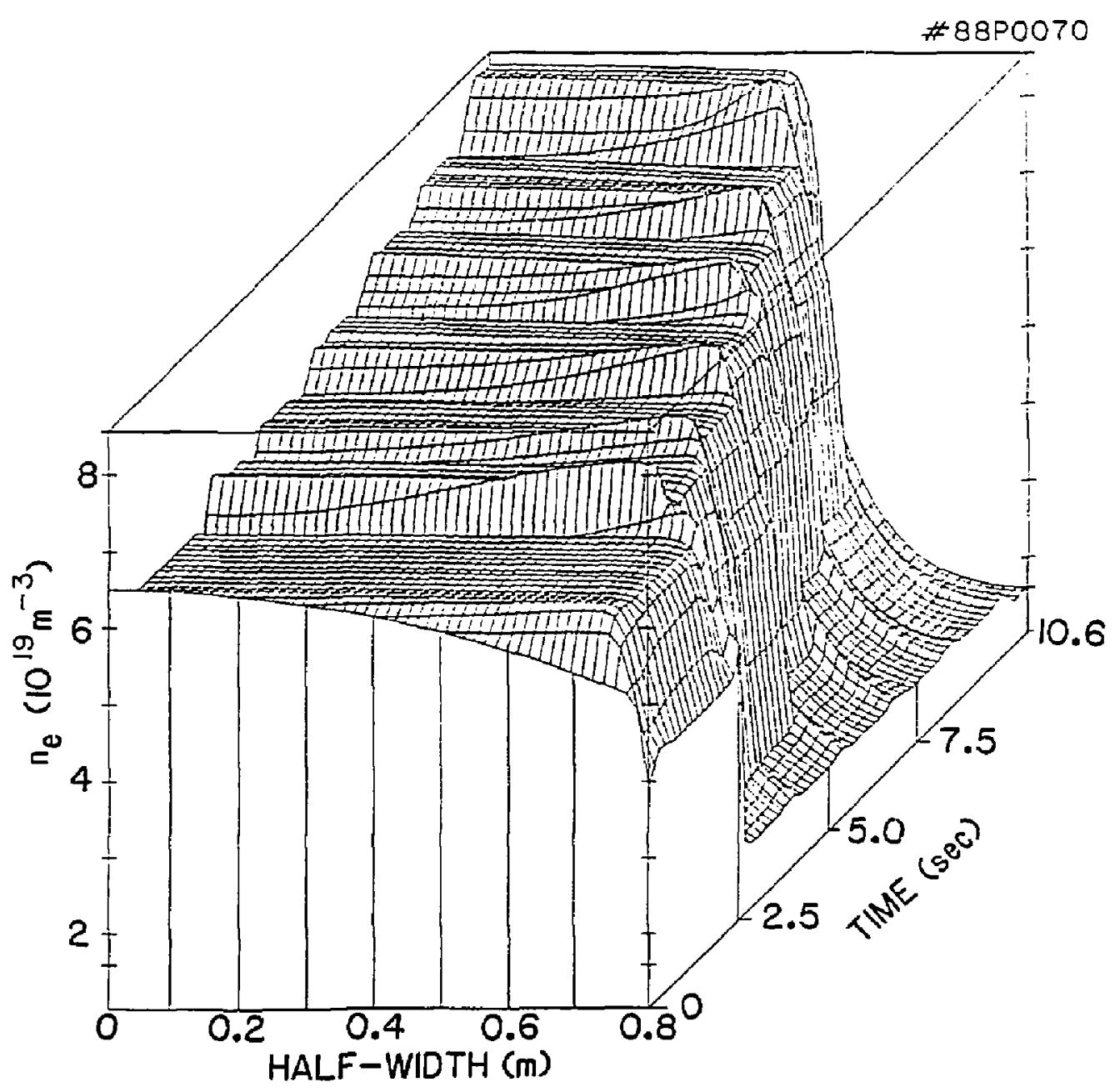

EIG. 7 


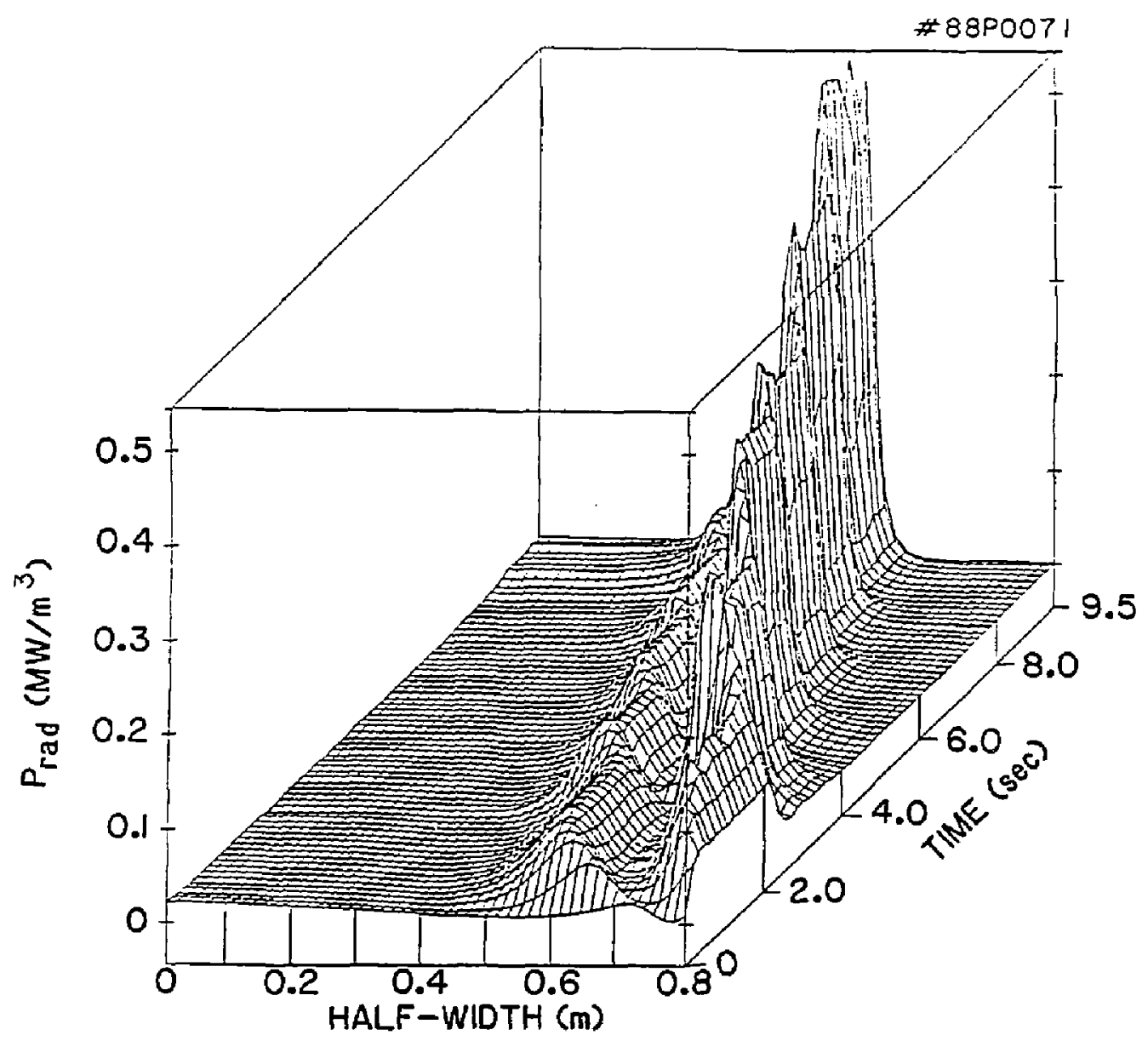

FIG. 8 


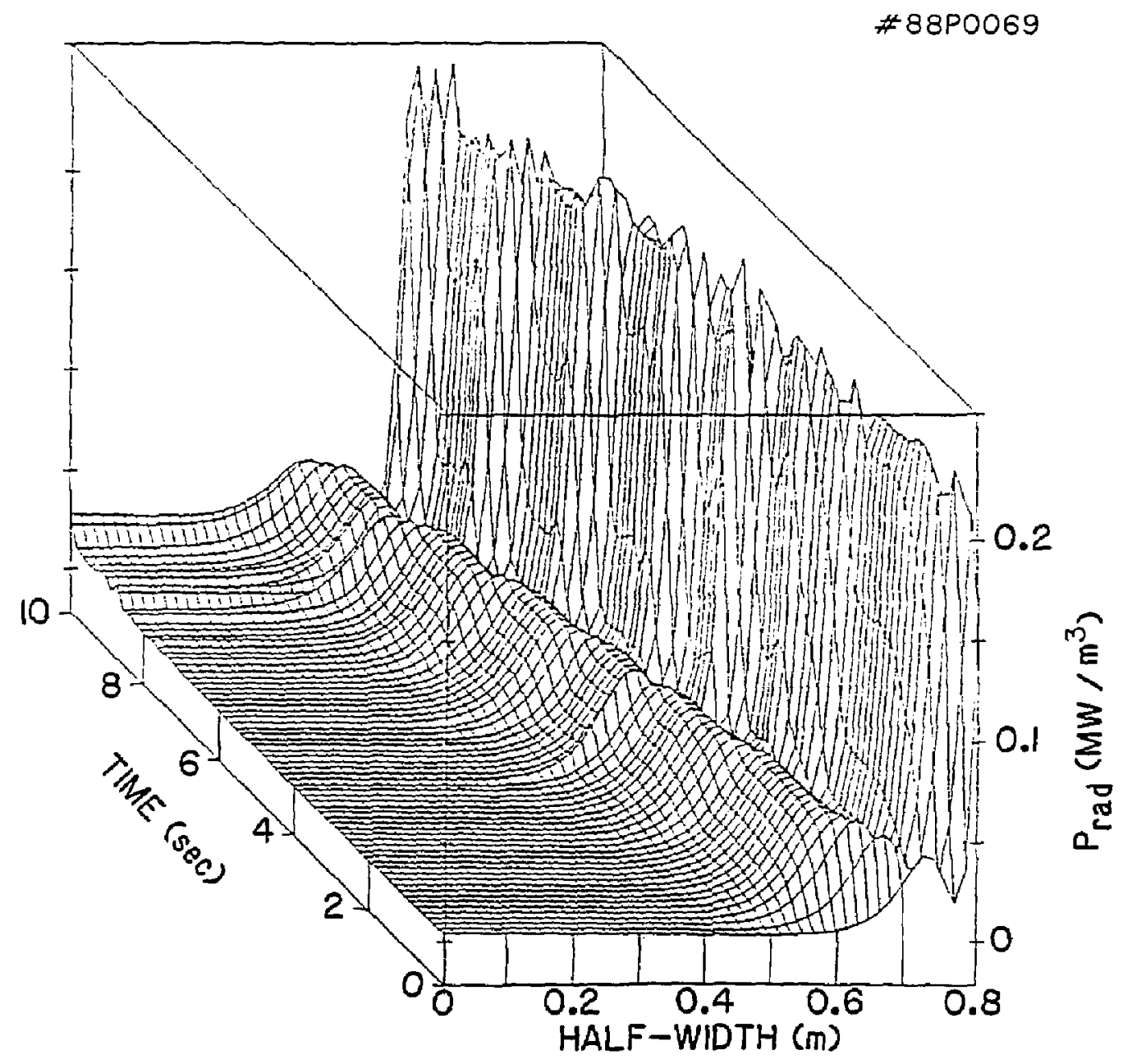

FIG. 9 
Dr. Frank J. Paolonj, Univ of Wollongang, AUSTRALIA Prof. M, H. Brennan, Univ Syaney, AuSTRALIA PIasna Research Lab., Australian Nat. Univ., AUSTRALIA Prof, I.R. Jones, FI inders Univ., AUSTRALIA

Prot. F, Cap, Inst Theo Phys, AUSTRIA

Prof. M. Heicdler, Institut $f$ ur Theoretische Physik, AUSTRIA M. Goossens, Astronomiseh Instituut, BELGIUM Ecole Royale Militairo, Lab de Phys Plasmas, AELGIUM Comission-European, Dg-X $\mid$ I Fusion Prog, BELGIUM Prof. R. Bouciqua, Laboratorium voor Natuurkunde, BELGIUM Dr. P,H. Sakanaka, Instituto Fisica, BRAZIL instituto De Pesquisas Espaciast-INPE, BRAziL Documents office, Atomic Energy of Canada Limited, CAMADA Dr. M.P. Bachynski, MPB Tochnologies, inc., CANADA Dr. H.M. Skarsgard, University of Saskatchewan, CANADA Dr. H. Barnard, University of British Calumbia, Canada Prof. J. Teichmann, Univ, of Montred, CANADh Prof. S.R. Sroenivasan, University of Calgary, CANADA Prof. Tudor W. Johnston, INRS-Energi i , CANADA Dr. C.R. James, Univ. of Alborta, CANADA Or. Peter Lukac, Komenskeho Univ, CZECHOSLOVAKIA The Librarian, Culhan Laboratory. ENGLAND The Librarian, Rutherford ADpleton Laboratory, ENGLANO Mrs. S.A. Hutchinson, JET Library, ENGLANO c. Mouttet, Lab. de Physique des Milieux lon is es, FRance J. Radet, CEN/CADARACHE - Bat 506, FRANCE

Univ, of loannina, Library of Physics Dept. GReECE Or. Tom Mual, Academy Bibliographic Ser., Hong KONG Prearint Library, Hungarian Academy of Sciences, HUNGARY Dr. B. Dasgupta, Saho Inst of Nucl. Phys., INOIA Dr. P. Kaw, Institute for PIasma Research, INDIA Dr. Philip Rosenau, Israel Inst. Tech, ISRAEL Librarian, Int'| Ctr Theo Phys, ITALy Prof. G. Rostagni, Univ Di Padova, ITALY Miss clelia de Paio, Assoc EuRATOM-EnEA, Italy Biblioteca, Instituto di fisica del Plasma, I TALY Or. H. Yamato, Toshiba Ros \& Dav, JAPAN Proł. 1. Kawakemi, Atomic Energy Res. Instituto, JAPAN Prot. Kyoji Nishikawa, Univ of Hiroshima, JAPAN Direc. Dept. Large Jokamak Res. JAER!, JAPAN Prof. Satoshi Iton, Kyushu University, JAPAN Research Info Center, Nagoya University, JAPAN Prof. S. Tanaka, Kyoto University, JAPAN Library, Kyoto University, JAPAN

Prof, Nobuyuki Inoue, University of TOKYO, JAPAN 5. Mori, JAERI, JAPAN Librarian, Korea Advanced Energy Res. Institute, KOREA Prof. O.l. Choi, Adv. Inst Sci \& Tech, KOREA Prof. 6.5. Liley, University of Walkato, NEW ZEALAND Institute of Plasma Physics, PEOPLE's REPUBLIC OF CHINA Librarian, Institute of Phys., PEOPLE'S REPUBLIC OF CHINA Library, Tsing Hua Univarsity, PEOPLE's REPUBLIC OF CHINA
Z. Li, Southwest Inst. Physies, PEOPLE'S REPUELIC of CHINA Prof. J.A.C. Cabral, fnst Suderior Tecnico, PORTUGAL Dr. Dctavian Potrus, AL I CUZA University, ROMANIA Dr. Johan de Villiers, Fusion Studies, AEC, SO AFRICA Prof. M.A. Hellberg, University of Natal, SO AFRICA C.I.E.M.A.T., Fusion Div. Library, SPAIN Dr. Lennart Stentlo, Un iversity of UMEA, SWEDEN Library, Royal Inst Tech, SWEDEN

Prot. Hans Wilheimson, Chalmers Univ Tech, SWEDEN Centre Phys des Plasmas, Ecole Polytech Fed, SWITZERLAND Bibliotheek, fom-Inst Voor PIasma-Fysica, THE NETHERLANDS Dr. D.D. Ryutov, Sibarian Acad Sci, USSR

Dr. G.A. El i seev, Kurchatov Institute, USSR

Or. Y.A. Glukhikh, Inst Electrophysical Apparatus, USSR

Or. Y.T. Tolok, Inst. Phys. Tech. USSA

Dr. L.M, Kovrizmnykh, Institute Gen. Physies, USSR Nuejear Res. Estabtishment, Julich Ltd., W. GERMANY Gibliothek, Inst. Fur Plasmaforschung, W. GEAMANY Dr. K. Senindler, Ruhr Universitot Bochum, W. GEFuMANY ASDEX Raading Rm, IPP/Max-Planck-Institut fur

Plasmaphysik. W. GERMANY

Librarian, Max-PIanck Institut, W. GERMANY

Prof. R.K. Janev, Inst Phys, YugosLAyia 